\title{
Statistical Indentation Techniques for Hydrated Nanocomposites: Concrete, Bone, and Shale
}

\author{
Franz-Josef Ulm, ${ }^{\dagger}$ Matthieu Vandamme, Chris Bobko, and Jose Alberto Ortega \\ Department of Civil and Environmental Engineering, Massachusetts Institute of Technology, Cambridge, \\ Massachusetts 02139 \\ Kuangshin Tai and Christine Ortiz
}

Department of Materials Science and Engineering, Massachusetts Institute of Technology, Cambridge, Massachusetts 02139

Concrete, bone and shale have one thing in common: their loadbearing mineral phase is a hydrated nanocomposite. Yet the link between material genesis, microstructure, and mechanical performance for these materials is still an enigma that has deceived many decoding attempts. In this article, we advance statistical indentation analysis techniques that make it possible to assess, in situ, the nanomechanical properties, packing density distributions, and morphology of hydrated nanocomposites. These techniques are applied to identify intrinsic and structural sources of anisotropy of hydrated nanoparticles: calcium-silicatehydrate $(\mathrm{C}-\mathrm{S}-\mathrm{H})$, apatite, and clay. It is shown that $\mathrm{C}-\mathrm{S}-\mathrm{H}$ and apatite, the binding phase in, respectively, cement-based materials and bone, are intrinsically isotropic; this is most probably due to a random precipitation and growth process of particles in calcium oversaturated pore solutions, which can also explain the nonnegligible internanoparticle friction. In contrast, the load-bearing clay phase in shale, the sealing formation of most hydrocarbon reservoirs, is found to be intrinsically anisotropic and frictionless. This is indicative of a 'smooth' deposition and compaction history, which, in contrast to mineral growth in confined spaces, minimizes nanoparticle interlocking. In all cases, the nanomechanical behavior is governed by packing density distributions of elementary particles delimitating macroscopic diversity.

\section{Introduction}

$\mathrm{H}$ YDRATED nanocomposites are a class of complex chemomechanical materials that possess a high degree of

D. Green - contributing editor

\footnotetext{
Manuscript No. 23278. Received May 29, 2007; approved July 23, 2007.

This work was financially supported by (1) the Lafarge Corporation for C-S-H research, (2) the US Army through the MIT Institute for Soldier Nanotechnologies for bone research, and (3) the MIT-OU GeoGenome Industry Consortium for shale research. Further support was provided by Ecole National des Ponts et Chaussees (France), enabling M. Vandamme's doctoral studies at MIT.

Author to whom correspondence should be addressed. e-mail: ulm@mit.edu
}

heterogeneity from atomistic scales to the macroscopic scales. To name a few, this class of materials includes the calciumsilicate-hydrates $(\mathrm{C}-\mathrm{S}-\mathrm{H})$, the binding phase in all cementitious materials; the load bearing clay fabric in shale, the sealing formation in most hydrocarbon reservoirs; and hydroxyapatite, the mineral binding phase of bone's ultrastructure. All these materials have in common the presence of structural water incorporated into an often plate- or sheet-like arrangement of atoms at nanoscales, which justifies their name as hydrated nanocomposites. These hydrated nanoparticles form the fundamental building block whose behavior is expected to delimitate macroscopic material diversity. However, the link between composition, microstructure, and mechanical performance of hydrated nanocomposites is still an enigma that has deceived many decoding attempts from experimental and theoretical sides. The focus of this paper is to advance emerging techniques that make it possible to assess in situ the nanoproperties of such highly heterogeneous hydrated nanocomposites, and to identify common features of such materials at nano- and microscales.

The paper is structured as follows: the first part is devoted to novel nanoexperimental methods and analysis that allow probing mechanically the nanofabric of hydrated nanocomposites. The method we introduced for measuring hardness and elastic properties of highly heterogeneous materials by a statistical analysis of instrumented indentation techniques has widely been adopted and used in the characterization of nanomechanical behavior of $\mathrm{C}-\mathrm{S}-\mathrm{H},{ }^{1-4}$ shales, ${ }^{5,6}$ and bones. ${ }^{7}$ Its attractiveness stems largely from the fact that properties of mechanically meaningful phases can be identified in situ by performing large grids of indentations on highly heterogeneous samples, with a proper choice of the indentation depth to ensure the self-similar properties of classical continuum indentation analysis. ${ }^{8,9}$ For this reason, the method is most suited for hydrated nanocomposites, whose multiscale material phases cannot be recapitulated in bulk form, and for which it is difficult to indent on a specific material phase with sufficient repeatability. In the first part of this paper, we also present the latest experimental protocol we developed and validated for different hydrated nanocomposites.
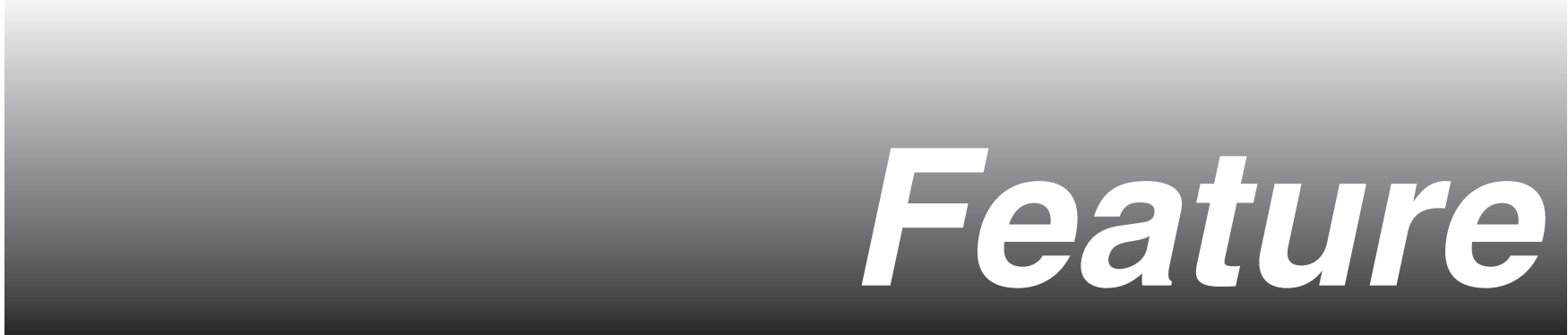
The second part is devoted to the application of this protocol to various hydrated nanocomposites: $\mathrm{C}-\mathrm{S}-\mathrm{H}$, clay, and apatite. In particular, from scaling the strength and stiffness properties of these different hydrated nanocomposites with their packing density, we aim at identifying intrinsic and structural sources of anisotropy of hydrated nanocomposites.

\section{Experimental Procedure}

While the use of instrumented nanoindentation has been extensively studied in the past two decades (for recent reviews, see, Oliver and Pharr ${ }^{8}$, and Cheng and Cheng ${ }^{9}$ ), the tools have been limited, until recently, to either homogeneous samples or to layered samples (thin films) whose thicknesses are previously known. ${ }^{10}$ Recently developed and refined techniques, however, have extended the application of nanoindentation to heterogeneous, composite materials. ${ }^{1,3,11}$

\section{(1) Indentation in Homogeneous Materials}

Briefly, we recall that nanoindentation consists of making contact between a sample and an indenter tip of known geometry and mechanical properties, followed by a continuously applied and recorded change in load, $P$, and depth, $h$. Typical tests consist of a constantly increasing load, followed by a short hold and then a constant unloading; a $P-h$ curve is reported. The analysis of the $P-h$ curve proceeds by applying a continuum scale model to condense the indentation response into two indentation properties; indentation modulus, $M$ :

$$
M=\frac{\sqrt{\pi}}{2} \frac{S}{\sqrt{A_{\mathrm{c}}}}
$$

and indentation hardness, $H$ :

$$
H=\frac{P}{A_{\mathrm{c}}}
$$

where $S=\mathrm{d} P / \mathrm{d} h$ is the (measured) initial slope of the unloading branch of the $P-h$ curve, $P$ is the (measured) maximum indentation load, and $A_{\mathrm{c}}$ is the projected contact area of the indenter on the sample surface (Fig. 1). The projected contact area, $A_{\mathrm{c}}$, is typically determined as a function of the (measured) maximum indentation depth, $h_{\text {max. }}$.

Furthermore, the two indentation properties $(M, H)$ can be linked to the elastic and strength properties of the indented material. In the isotropic case, $M$ reduces to the plane-stress modulus: ${ }^{13,14}$

$$
M=\frac{E}{1-v^{2}}=\frac{C_{11}^{2}-C_{12}^{2}}{C_{11}}
$$

where $E$ is the Young's modulus, $v$ the Poisson's ratio; $C_{11}=C_{1111}$ and $C_{12}=C_{1122}$ are the two stiffness coefficients describing the isotropic elastic behavior of the half-space. In the case of anisotropic elasticity, things are more complicated as the indentation modulus depends on the direction of indentation w.r.t. the material's symmetry axis. General solutions for this problem are quite involving, ${ }^{15-19}$ but can be simplified into explicit relations for specific material symmetries with high accuracy. For instance, in the case of a transverse isotropic elastic half space, with $x_{3}$ being the material symmetry axis, the indentation moduli $M_{i}=M\left(x_{i}\right)$ obtained from indentation in the principal material axis $x_{i}$ are linked to the five independent elasticity constants by ${ }^{20}$

$$
\begin{aligned}
M_{3} & =M\left(x_{3}\right) \\
& =2 \sqrt{\frac{C_{11} C_{33}-C_{13}^{2}}{C_{11}}\left(\frac{1}{C_{44}}+\frac{2}{\sqrt{C_{11} C_{33}}+C_{13}}\right)^{-1}}
\end{aligned}
$$

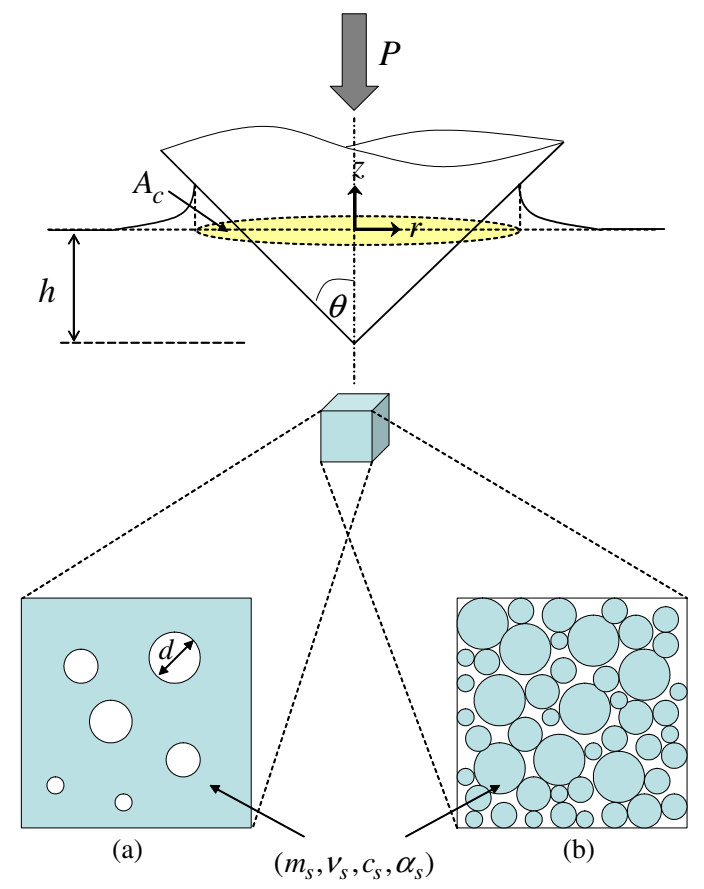

Fig. 1. Conical indentation in a porous material, composed of a solid phase and pore space: (a) matrix-porosity morphology; (b) perfectly disordered, polycrystal morphology [adapted from Cariou et al. ${ }^{12}$ ].

$$
M_{1}=M\left(x_{1}\right)=M\left(x_{2}\right)=\sqrt{\sqrt{\frac{C_{11}}{C_{33}}} \frac{C_{11}^{2}-C_{12}^{2}}{C_{11}} M_{3}}
$$

where we employ Voigt notation $C_{11}=C_{1111}, C_{12}=C_{1122}$, $C_{13}=C_{1133}, C_{33}=C_{3333}, C_{44}=C_{2323}=C_{1313}$.

\section{(2) Indentation in Heterogeneous Materials: Grid- Indentation Technique}

Recognizing the high heterogeneity of hydrated nanocomposites at the nano- and microscale, application of the indentation technique is a challenge, as it is difficult to choose to indent on a specific material phase with sufficient repeatability. To address this challenge, it is advantageous to perform large grids of indentations on heterogeneous samples. Then, if the grid size and indentation depth are chosen properly, each indentation test may be treated as an independent statistical event; and statistical techniques may be applied to analyze the results.

To illustrate our purpose, consider the following thought experiment:

Consider an infinite half-space composed of two materials of different mechanical properties. Provided that the indentation depth is much smaller than the characteristic size of the two phases, a random indentation on the sample's surface provides access to either phase properties, with a probability that equals the surface fraction the two phases occupy on the sample surface. By contrast, an indentation to a depth much larger than the characteristic size of the two phases samples mechanically the composite response.

This thought-experiment illustrates how the classical tools of instrumented indentation can be extended to heterogeneous materials, through a careful choice of the indentation depth that depends on the size of elementary component. It calls for a statistical analysis of a large array of indentation tests and a subsequent statistical deconvolution of the indentation results.

The deconvolution technique begins with the generation of the experimental cumulative distribution function (CDF). Let $N$ be the number of indentation tests performed on a specimen, and $\left\{M_{i}\right\}$ and $\left\{H_{i}\right\}(i=1, N)$ the sorted values of the measured 
indentation modulus $M$ and indentation hardness $H$. The $N$ points of the experimental CDF of $X=(M, H)$, denoted by $D_{X}$, are obtained from

$$
D_{X}\left(X_{i}\right)=\frac{i}{N}-\frac{1}{2 N} \quad \text { for } i \in[1, N], \quad X=(M, H)
$$

Once the experimental CDFs are known, the form of the model CDFs are specified. Consider the heterogeneous material to be composed of $j=1, n$ material phases with sufficient contrast in mechanical phase properties. Each phase occupies a surface fraction, $f_{j}$, of the indented surface. The distribution of the mechanical properties of each phase is assumed to be correctly approximated by Gaussian distributions, identified by the mean values $\mu_{j}^{M}$ and $\mu_{j}^{H}$ and the standard deviations $s_{j}^{M}$ and $s_{j}^{H}$, of the indentation modulus $M$ and the indentation hardness $H$, respectively. The CDF for each (Gaussian distributed) phase is given by

$$
\begin{aligned}
j= & 1, n, D\left(X_{i} ; \mu_{j}^{X}, s_{j}^{X}\right) \\
= & \frac{1}{s_{j}^{X} \sqrt{2 \pi}} \\
& \times \int_{-\infty}^{X_{i}} \exp \left(\frac{-\left(u-\mu_{j}^{X}\right)^{2}}{2\left(s_{j}^{X}\right)^{2}}\right) \mathrm{d} u, \quad X=(M, H)
\end{aligned}
$$

The $n \times 5$ unknowns $\left\{f_{j}, \mu_{j}^{M}, s_{j}^{M}, \mu_{j}^{H}, s_{j}^{H}\right\}, j=1, n$ are determined by minimizing the difference between the experimental
CDFs and the weighted model-phase CDFs:

$$
\begin{gathered}
\min \sum_{i=1}^{N} \sum_{X=(M, H)}\left(\sum_{j=1}^{n} f_{j} D\left(X_{i} ; \mu_{j}^{X}, s_{j}^{X}\right)-D_{X}\left(X_{i}\right)\right)^{2} \\
\text { s.t. } \\
\sum_{j=1}^{n} f_{j}=1
\end{gathered}
$$

where the constraint of the minimization problem requires that the surface fractions of the different phases sum to one. To ensure that phases have sufficient contrast in properties, and thus to avoid the overlap of two neighboring Gaussians, the optimization problem is additionally constrained by

$$
\mu_{j}^{X}+s_{j}^{X} \leq \mu_{j+1}^{X}+s_{j+1}^{X}, \quad X=(M, H)
$$

The results of the deconvolution technique are estimates of the mean and standard deviation of indentation modulus and hardness for each mechanical phase, and the surface fraction. For randomly organized materials, the surface fractions are actually volume fractions.

The deconvolution technique here proposed differs from the original deconvolution technique we suggested earlier, ${ }^{3,4}$ in that it is analytically more convenient to deconvolute the CDF rather than the probability density function (PDF), because generation of the experimental PDF requires a choice of bin-size for histogram construction.

By way of illustration, Fig. 2 shows the results of the deconvolution technique in terms of both the CDFs and the PDFs for indentation modulus and indentation hardness for a white cement paste, prepared at a water:cement ratio of $w / c=0.5$. The PDFs, which are more physically intuitive,
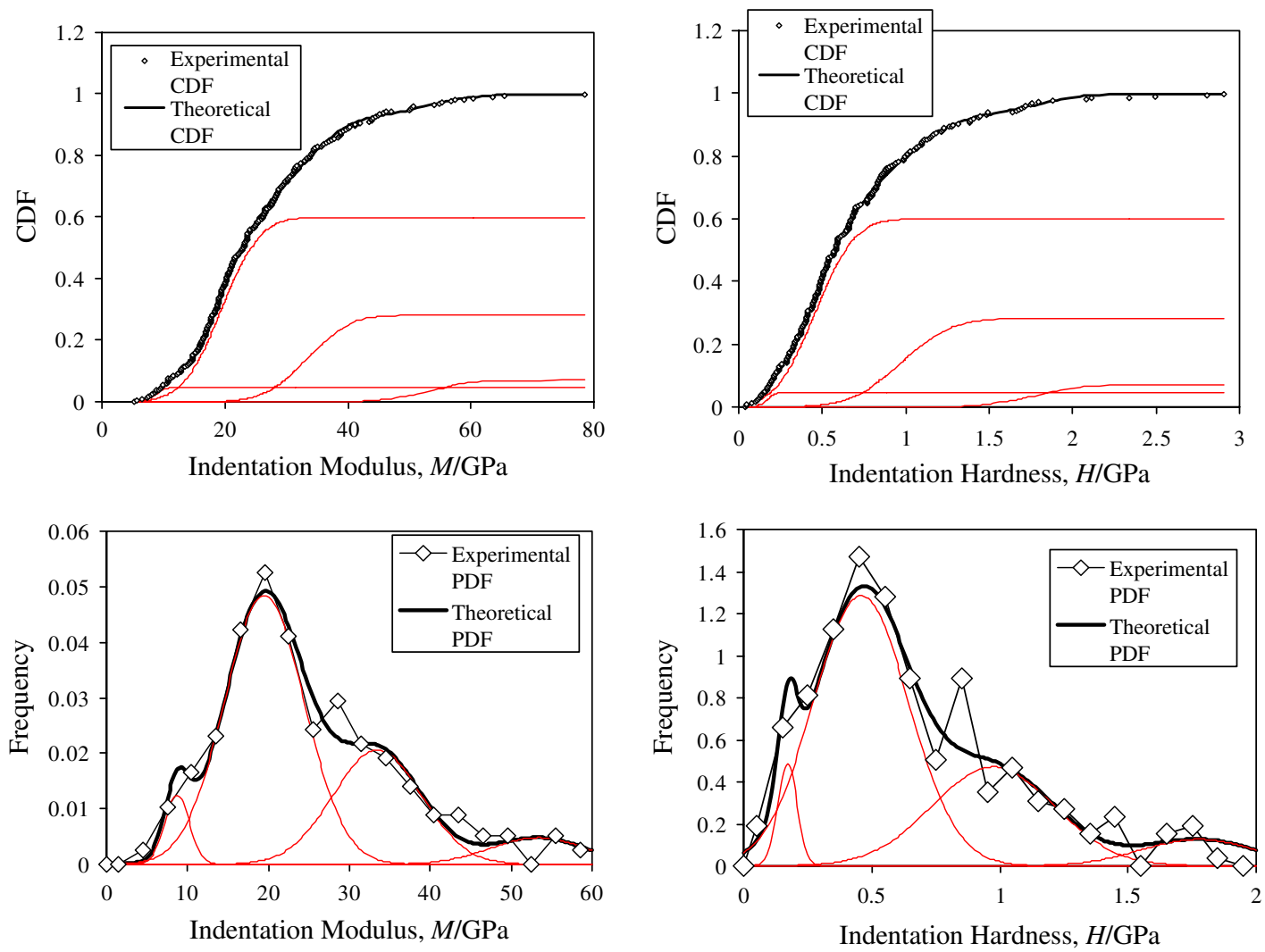

Fig. 2. Statistical Indentation Analysis of a $w / c=0.5$ white cement paste: cumulative distribution functions (CDF-top) and probability density functions (PDF-bottom) of indentation modulus $M$ (left) and indentation hardness $H$ (right). The experimental CDF is constructed using 300 ( $M, H$ ) data points. It is deconvoluted in a series of four phase-specific CDFs, which are assumed to be Gaussian, and which are also displayed. 
Table I. Deconvolution Results of 300 Indentation Tests of a $w / c=0.5$ White Cement Paste

\begin{tabular}{|c|c|c|c|c|c|c|c|}
\hline Phase $j$ & \multicolumn{2}{|c|}{$M(\mathrm{GPa})$} & \multicolumn{2}{|c|}{$H(\mathrm{GPa})$} & $\begin{array}{c}\text { Surface/volume fraction } \\
f_{i}\end{array}$ & \multicolumn{2}{|c|}{ Packing density } \\
\hline 1 & 8.71 & 1.51 & 0.17 & 0.04 & 0.05 & 0.547 & 0.020 \\
\hline 3 & 33.44 & 5.45 & 0.97 & 0.24 & 0.28 & 0.746 & 0.047 \\
\hline 4 & 53.36 & 5.85 & 1.78 & 0.22 & 0.07 & 0.859 & 0.037 \\
\hline
\end{tabular}

$M$, indentation modulus; $H$, indentation hardness.

show that two major and two minor hydration products are present in the hydrated material system, which are sensed by nanoindentation. The mechanical properties of those phases are listed in Table I. For cement-based materials, the two major hydration phases that make up, in a $w / c=0.5$ material, close to $90 \%$ of all data have been previously attributed to coincide with the low-density $\mathrm{C}-\mathrm{S}-\mathrm{H}$ phase ( $\mathrm{LD} \mathrm{C}-\mathrm{S}-\mathrm{H}$ ) and the high-density $\mathrm{C}-\mathrm{S}-\mathrm{H}$ phase (HD C-S-H) ${ }^{1-3,21}$ This was established on the basis of average $\mathrm{C}-\mathrm{S}-\mathrm{H}$ porosity values reported by Jennings. ${ }^{22}$ The next section shows how the same result can be directly obtained from nanoindentation by a consistent micromechanical modeling of the indentation response, linking $(M, H)$ to the microstructure of the different hydration phases.

\section{(3) Micromechanics Link Between Indentation Response and Microstructure}

Consider an indentation test in a porous material composed of a cohesive-frictional solid phase (volume fraction $\eta$ ) and an (empty) pore space (volume fraction $\varphi_{0}=1-\eta$ ); Fig. 1. For scale separability reasons, ${ }^{23,24}$ the characteristic size of the porosity, say $d$, is assumed to be much smaller than the indentation depth $h$. As a result, the indentation modulus $M$ and the indentation hardness $H$, which are extracted from the indentation test, are composite properties, representative of the homogenized response of the porous material. In a dimensionless form, the homogenized response is formally written as

$$
\begin{aligned}
& \frac{M}{m_{s}}=\Pi_{M}\left(v_{s}, \eta, \eta_{0}\right) \\
& \frac{H}{c_{s}}=\Pi_{H}\left(\alpha_{s}, \eta, \eta_{0}, \theta\right)
\end{aligned}
$$

These expressions recognize that the homogenized response depends on (at least) the following four sets of parameters:

(1) Intrinsic solid properties, which for an isotropic material are the asymptotic contact modulus $m_{s}=\lim _{\eta=1} M$ and the solid's Poisson's ratio $v_{s}$, cohesion $c_{s}$ and friction coefficient $\alpha_{s}$.

(2) The solid fraction or packing density $\eta$ ('one minus porosity').

(3) Microstructural morphology parameters, namely the solid percolation threshold, $\eta_{0}$, that is the solid fraction required to provide a continuous force path through the system. Indeed, a porous material that possesses a clear matrix-porosity morphology has a continuous solid phase for any packing density $\eta_{0}=0<\eta \leq 1$ (Fig. 1(a)). In contrast, a perfectly disordered porous material system (Fig. 1(b)) has a solid percolation threshold of $\eta_{0} \simeq 1 / 2$, below which the solid becomes unstable. ${ }^{25}$ In between these limit cases, any order introduced to the system (for instance, particle shape $\mathrm{e}^{26,27}$ and/or connectiveness between particles) decreases the percolation threshold from $\eta_{0} \simeq 1 / 2$ toward $\eta_{0}=0$.

(4) Some indenter geometrical-specific parameters, represented here by the cone angle $\theta$. In the case of the most commonly employed indenter shape, the three-sided Berkovich indenter, which possesses the same scaling behavior as conical indenters, ${ }^{28} \theta=70.32^{\circ}$, is the equivalent half-cone angle. Vary- ing the cone angle in indentation of cohesive-frictional materials has been successfully used to determine the friction angle from indentation data. ${ }^{7,29}$

Hydrated nanocomposites, namely $\mathrm{C}-\mathrm{S}-\mathrm{H}$, hydroxyapatite in bones, and clays, possess a very distinct disordered morphology of the solid phase, similar to a polycrystal..$^{3,5-7,30,31}$ For such a disordered porous material system, linear micromechanics provides the following relations for the dimensionless indentation modulus function $(10)^{3}$ :

$$
\Pi_{M}\left(r_{s}, \eta, \eta_{0}=1 / 2\right)=\mathcal{G} \frac{\left(9 \eta r_{s}+4 \mathcal{G}+3 r_{s}\right)\left(3 r_{s}+4\right)}{4\left(4 \mathcal{G}+3 r_{s}\right)\left(3 r_{s}+1\right)}
$$

where $r_{s}=2\left(1+v_{s}\right) / 3\left(1-2 v_{s}\right)>0$, and $\mathcal{G}$ is the composite shear-to-solid shear moduli ratio:

$$
\begin{aligned}
\mathcal{G}= & \frac{1}{2}-\frac{5}{4}(1-\eta)-\frac{3}{16} r_{s}(2+\eta)+\frac{1}{16} \\
& \times \sqrt{144\left(1-r_{s}\right)-480 \eta+400 \eta^{2}+408 r_{s} \eta-120 r_{s} \eta^{2}+9 r_{s}^{2}(2+\eta)^{2}}
\end{aligned}
$$

For a Poisson's ratio $v_{s}=1 / 5$, Eq. (12) reduces to a linear scaling of the indentation modulus with the packing density $\Pi_{M}=2 \eta-1 \geq 0$.

Based on nonlinear micromechanics, ${ }^{24}$ similar relations were recently developed for the scaling (11) of the indentation hardness with the packing density ${ }^{12}$

$$
\begin{aligned}
\Pi_{H}\left(\alpha_{s}\right)= & \Pi_{H}(0) \\
& \times\left[1+(1+\eta) \alpha_{s}-(d-e \eta) \alpha_{s}^{2}-(f-g \eta) \alpha_{s}^{5}\right]
\end{aligned}
$$

where $\Pi_{H}(0)=\Pi_{H}\left(\alpha_{s}=0\right)$ is the frictionless portion of the function given by

$$
\Pi_{H}(0)=\frac{12 \eta(a-b \eta) \sqrt{(2 \eta-1)(2+\eta)}}{(1-c \eta)(2+\eta)}
$$

and where $a=0.19567, b=0.03739, c=0.77999, d=20.3138$, $e=31.5352, f=52.1817$, and $g=99.3465$ are all constants associated with the Berkovich indenter geometry and a polycrystal morphology with a percolation threshold of $\eta_{0}=1 / 2$.

In a forward application, use of the scaling relations (10) and (11) requires knowledge of four solid properties $\left(m_{s}, v_{s}, c_{s}, \alpha_{s}\right)$ and of the solid's packing density $\eta$ to determine the composite indentation quantities $M$ and $H$. In an inverse application, $N \geq 4$ indentation tests are required, to determine from experimental $(M, H)$ values the solid properties $\left(m_{s}, v_{s}, c_{s}, \alpha_{s}\right)$ and the solid's packing density $\eta$. Therefore, provided the existence of a unique solid phase present in the porous microstructure, the scaling relations are a versatile tool to probe the microstructure sensed by the large array of grid indentation tests.

The hypothesis testing approach can be sketched as follows:

(1) Hypothesis: Assume the existence of a unique solid phase of the porous material. 

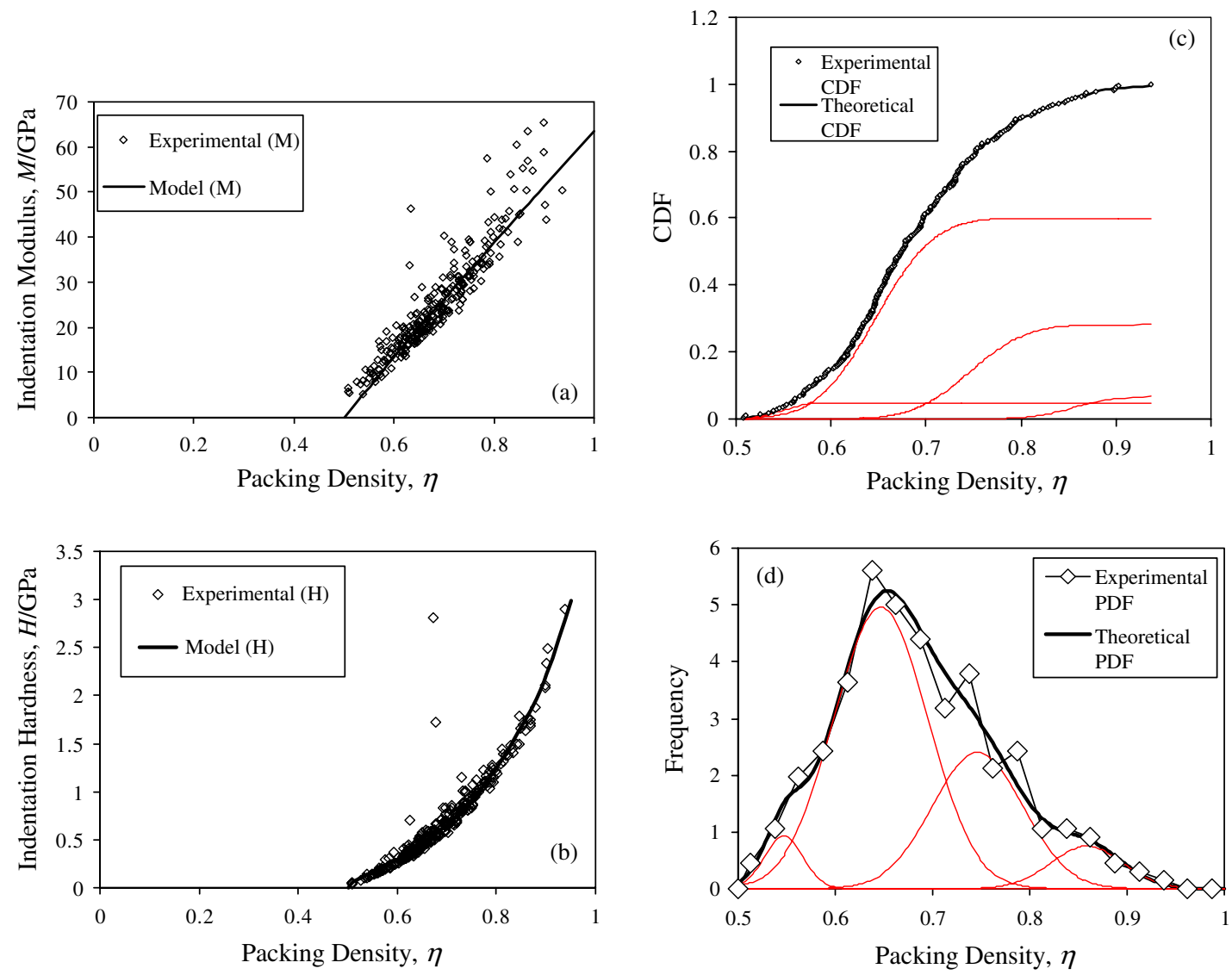

Fig. 3. Statistical indentation analysis of packing density distribution of a $w / c=0.5$ white cement paste: packing density scaling relations of (a) indentation modulus $M$ and (b) indentation hardness $H$. Deconvolution of packing density distribution: (c) cumulative distribution functions (CDF), and $(d)$ derived probability density functions (PDF). The deconvolution results show that there are two major and two minor hydration phases present in the cement paste, which differ in the C-S-H packing density.

(2) Calibration: From $N \gg 4$ indentation tests, determine the four unique solid properties and the $N$ packing densities, by minimizing the quadratic error between the experimental values $X=(M, H)$ and the theoretical scaling relations:

$$
\min _{\substack{\kappa_{S}^{S}=\left(m_{s}, c_{s}\right) \\ \chi_{X}^{X}=\left(v_{s}, \alpha_{s}\right) \\ \eta_{i}=1, N}} \sum_{i=1}^{N} \sum_{X=(M, H)}\left(1-\frac{\kappa_{X}^{S} \Pi_{X}\left(\chi_{X}^{S}, \eta_{i}\right)}{X_{i}}\right)^{2}
$$

By way of illustration, Figs. 3(a) and (b) show the results of the minimization procedure in form of $M(\eta)$ and $H(\eta)$ scaling plots for the 300 white cement paste nanoindentation tests considered before. The mean relative error of the minimization is $\bar{e}_{M}=$ $-4.0 \%$ for indentation modulus, and $\bar{e}_{H}=0.1 \%$ for the indentation hardness. The standard deviation of the relative error, which is a measure of the data dispersion, is $\overline{\bar{e}}_{M}=17.3 \%$ for indentation modulus and $\overline{\bar{e}}_{H}=13.1 \%$ for indentation hardness. The fitted solid properties $\left(m_{s}, v_{s}, c_{s}, \alpha_{s}\right)$, assumed to be the same in all indentation tests, are summarized in Table II.

(3) Deconvolution: Use the three indentation test specific quantities $Y=(M, H, \eta)$ for deconvolution analogous to (8)

$$
\min \sum_{i=1}^{N} \sum_{Y=(M, H, \eta)}\left(\sum_{j=1}^{n} f_{j} D\left(Y_{i} ; \mu_{j}^{Y}, s_{j}^{Y}\right)-D_{Y}\left(Y_{i}\right)\right)^{2}
$$

where $D_{Y}\left(Y_{i}\right)$ are the experimental CDFs of form (6) constructed from the sorted values of $\left\{Y_{i}\right\}=\left(\left\{M_{i}\right\},\left\{H_{i}\right\}\right.$, $\left.\left\{\eta_{i}\right\}\right) ; i=1, N$. By way of illustration, Figs. 3(c) and (d) display the deconvolution results in terms of both the CDFs and the PDFs of the packing density. The addition of the packing density for deconvolution is found not to affect significantly the deconvolution of $X=(M, H)$; meaning - as expected from its very determination - that the packing density $\eta$ is not a statistically independent variable. In particular, a comparison of the PDFs displayed in Figs. 2 and 3(d) show some evidence that the different hydration phases in the white cement phase are structurally distinct through their packing density, yet compositionally similar through a unique set of solid properties (Table II).

(4) Confirmation: Confirm the packing density results by an independent technique. The particle packing density of different phases can be assessed by a variety of means, from a combination of mineralogy, density, and eventually porosity measurements. In the case of $\mathrm{C}-\mathrm{S}-\mathrm{H}$, we use measured mass density values provided by Jennings and colleagues, ${ }^{21,22}$ updated using

Table II. Mineral Properties of C-S-H Obtained by Fitting $300(M, H)$ Indentation Data of $w / c=0.5$ White Cement Paste to the Stiffness- and Hardness-Packing Density Relations

\begin{tabular}{lcc}
\hline C-S-H mineral properties & Set $^{\dagger} \# 1$ & Set $^{\dagger} \# 2$ \\
\hline Stiffness, $m_{s}(\mathrm{GPa})$ & 63.5 & 62.7 \\
Poisson's ratio, $v_{s}$ & -0.07 & 0.2 \\
Cohesion, ${ }^{\ddagger} c_{s}(\mathrm{GPa})$ & 0.336 & 0.354 \\
Friction coefficient, $^{\ddagger} \alpha_{s}$ & 0.269 & 0.254 \\
Friction angle, ${ }^{\S} \varphi$ (deg.) & 15.8 & 14.85 \\
\hline
\end{tabular}

In set 1, Poisson's ratio $v_{s}$ is a fitting parameter, while $v_{s}=1 / 5$ is fixed in set 2 . ${ }^{\ddagger}$ Cohesion and friction coefficient are to be understood in the sense of the Drucker-Prager strength model. ${ }^{\S}$ Friction angle is to be understood in the sense of the Coulomb material model, i.e. angle of repose. C-S-H, calcium-silicatehydrate. 
Table III. C-S-H Mass Density Reported by Jennings ${ }^{22}$ and Allen et al. ${ }^{32}$ at Different Scales; Derived Packing Density, and Comparison With Packing Density Distribution Obtained by Deconvolution Technique

\begin{tabular}{|c|c|c|c|c|}
\hline \multirow[b]{2}{*}{ Scale } & \multicolumn{2}{|c|}{$\operatorname{Density}^{\dagger}\left(\mathrm{kg} / \mathrm{m}^{3}\right)$} & \multicolumn{2}{|c|}{ Mean \pm SD } \\
\hline & $\rho_{\text {sat }}$ & $\rho_{\text {dry }}$ & Packing density ${ }^{\ddagger}$ & Packing density distribution ${ }^{\S}$ \\
\hline \multicolumn{5}{|l|}{$\mathrm{C}-\mathrm{S}-\mathrm{H}$ solid } \\
\hline Particle & \multicolumn{2}{|c|}{$2604^{\top}$} & & \\
\hline \multicolumn{5}{|l|}{ C-S-H composite } \\
\hline $\mathrm{LD} C-\mathrm{S}-\mathrm{H}^{\|}$ & 2050 & 1700 & $0.65 \pm 0.00$ & $0.65 \pm 0.05$ \\
\hline $\mathrm{HD} \mathrm{C}-\mathrm{S}-\mathrm{H}^{\|}$ & 2300 & 2000 & $0.73 \pm 0.05$ & $0.75 \pm 0.05$ \\
\hline
\end{tabular}

${ }^{\dagger} \rho_{\text {sat }}$ and $\rho_{\text {dry }}$ stand for the density respectively at full water saturation of the pore space and empty pore space. ${ }^{\dagger}$ Packing density from mass density values. ${ }^{\S}$ For comparison, values from packing density distribution for dominating hydration phase (from Table I). ${ }^{\bullet} \rho^{s}=2,604 \mathrm{~kg} / \mathrm{m}^{3}$ reported by Allen et al. ${ }^{32} \| \mathrm{Values}$ reported by Jennings et al. ${ }^{21} \mathrm{LD}$, low density; HD, high density; C-S-H, calcium-silicate-hydrate.

recently reported particle density values obtained from combining small-angle neutron and X-ray scattering data. ${ }^{32}$ In order to estimate the packing density, we take the mean of the saturated and the dry mass densities, $\rho_{\text {sat }}$ and $\rho_{\text {dry }}$, determined from

$$
\begin{aligned}
& \eta_{\text {sat }}=1-\frac{\rho_{\text {sat }}-\rho_{\text {dry }}}{\rho^{w}}, \quad \eta_{\text {dry }}=\frac{\rho_{\text {dry }}}{\rho^{s}} \\
& \eta=\frac{1}{2}\left(\eta_{\text {sat }}+\eta_{\text {dry }}\right)
\end{aligned}
$$

where $\rho^{w}$ and $\rho^{s}$ are the mass density of, respectively, the saturating fluid phase and the particle. Table III displays mass density and packing density values for the $\mathrm{LD} \mathrm{C}-\mathrm{S}-\mathrm{H}$ phase and the HD C-S-H phase. The values (almost) perfectly agree with the mean packing density values of the major hydration phases determined from a combination of micromechanics relations and the deconvolution of the nanoindentation data.

\section{(4) Some Remarks on Methods}

The statistical analysis of nanoindentation involves heavy error minimization procedures in both the $(M, H)$ fitting and the deconvolution of $(M, H, \eta)$. Such inverse analysis generally suffers from the non-uniqueness of the solution. A critical issue for inverse analysis is good start values for the error minimization. The following recommendations are made:

(1) Start values for error minimization (16): For the solid properties, mineral properties for $\kappa_{X}^{s}=\left(m_{s}, c_{s}\right)$, if available, while letting $\chi_{X}^{s}=\left(v_{s}=1 / 5, \alpha_{s}=0\right)$ are good start values for the backanalysis of the packing density using (16).

(2) Effect of Poisson's ratio: Among the four solid property parameters $\left(m_{s}, v_{s}, c_{s}, \alpha_{s}\right)$, the variability of the solid's Poisson's ratio $v_{s}$ shows a small effect on the stability of the inversion results. This is due to the fact that the Poisson's ratio is squared in the classical expression of isotropic indentation modulus (3). By way of illustration, Table II shows that the actual value of $v_{s}$ has little effect on the other fitted solid properties. Therefore, the error minimization (16) provides, at best, a trend whether the (isotropic) solid phase is rather highly compressible $v_{s} \leq 0$, or highly incompressible $v_{s}=1 / 2$.

(3) Extension to anisotropic elasticity: The error minimization (16) is based on the assumption of an isotropic nanosolid phase, whose behavior is condensed into the four solid property parameters $\left(m_{s}, v_{s}, c_{s}, \alpha_{s}\right)$. In the anisotropic elasticity case, for which the indentation modulus-stiffness relations are more complex (see Eqs. (4) and (5)), there are many more elasticity parameters to be considered that capture the anisotropy of the solid phase. This aspect is further developed here below.

\section{Some Applications}

By way of application, we show here below how the statistical indentation technique is applied in 'real' life indentation analysis of microstructural features of hydrated nanocomposites: $\mathrm{C}-\mathrm{S}-\mathrm{H}$, (anorganic) bone, and shales. In particular, the aim of this section is twofold: (1) to determine particle properties of highly heterogeneous materials, and (2) to show how this technique can be applied to discriminate intrinsic versus structural isotropy and anisotropy of such materials from nano-to-micro-scale contact experiments.

\section{(1) Intrinsic Isotropy of Randomly Oriented $\mathrm{C}-\mathrm{S}-\mathrm{H}$ Particles}

Despite the fact that the nanostructure of $\mathrm{C}-\mathrm{S}-\mathrm{H}$ is still not known, there is little doubt that the elementary $\mathrm{C}-\mathrm{S}-\mathrm{H}$ particle is anisotropic. Indeed, as it is known from its natural analogs, namely $1.4 \mathrm{~nm}$ tobermorite and jennite, crystalline $\mathrm{C}-\mathrm{S}-\mathrm{H}$ are atomically layered composites, ${ }^{33}$ in which a central $\mathrm{Ca}-\mathrm{O}$ sheet is flanked on both sides by rows of single dreierketten, which together with $\mathrm{Ca}$ atoms and water molecules form a pronounced layered structure. ${ }^{34}$ On the other hand, the 'real' C-S-H phase that forms during the hydration of Portland cement is poorly crystalline compared with tobermorite and jennite, and it is characterized by extensive atomic imperfections and structural variations at the nanometer scale. The question that therefore arises is whether these atomic imperfections cause the actual $\mathrm{C}-\mathrm{S}-\mathrm{H}$ particle, of elementary size of $5 \mathrm{~nm},{ }^{21,22,32}$ to be intrinsically isotropic or intrinsically anisotropic. The statistical indentation technique can address this question.

(A) Material and Surface Preparation: Cement paste cylinders of $10-\mathrm{mm}$ diameter were prepared at a water-cement ratio of $w / c=0.3$ using a cement from Le Teil (for the composition see Table IV). Following hydration, the sample was wrapped in aluminum until testing. For indentation testing, the same cylinder specimen was cut in two orthogonal directions of approximate thickness $5 \mathrm{~mm}$; one cut normal to the cylinder axis (noted as direction $x_{3}$ here below), the other parallel to the cylinder axis (noted as direction $x_{1}$ hereafter). The surface preparation follows a procedure described in detail in Bobko and Ulm, ${ }^{6}$ which minimizes both the roughness and sample disturbance of the highly heterogenous material: samples are mounted to stainless-steel plates for mounting in the indenter. They are then ground by hand on 45 grit diamond paper (with the help of a jig) to make the surface flat and parallel with the mounting plate. The samples are then polished with a $1-\mu \mathrm{m}$ diamond paste (oil-based) on TexMet pads (Buehler) mounted to a lapping wheel. From AFM testing, the RMS roughness obtained with this polishing procedure was $10 \mathrm{~nm}$, which comes quite close to a flat surface required for indentation testing.

(B) Indentation Test Parameters and Results: For each direction, 300 force-driven indentation tests to a maximum indentation force of $P=2.02 \pm 0.01 \mathrm{mN}$ were carried out. The load was increased linearly in $10 \mathrm{~s}$, kept at its maximum value for $5 \mathrm{~s}$, and decreased linearly in $10 \mathrm{~s}$. The average maximum indentation depth achieved with this protocol was the same for both indentation direction, $h=276 \pm 100 \mathrm{~nm}$. The fact that the same average depth is observed in orthogonal directions is a preliminary indication of an isotropic composition of the different material phases in the cement paste, which includes hydration products and unhydrated clinker in this low $w / c$ material. 
Table IV. Composition of the Cement from Le Teil of Lafarge in Mass Percentage of each Component Provided by the Manufacturer

\begin{tabular}{|c|c|c|c|c|c|c|}
\hline $\mathrm{CaO}$ & & $\mathrm{SiO}_{2}$ & $\mathrm{Al}_{2} \mathrm{O}_{3}$ & $\mathrm{Fe}_{2} \mathrm{O}_{3}$ & $\mathrm{SO}_{3}$ & LOI \\
\hline 67.17 & & 22.14 & 3.12 & 2.51 & 2.13 & 1.68 \\
\hline Alite & Belite & Ferrite & Aluminate & Anhydrite & Gypsum & Calcite \\
\hline 71.1 & 15.0 & 7.0 & 1.2 & 1.4 & 1.2 & 1.8 \\
\hline
\end{tabular}

Components with a mass percentage smaller than $1 \%$ are not included.

Our focus is on the properties of the hydration phases in the cement paste, requiring to separate the clinker phases from the data set. The $(M, H)$-packing density scaling provides an efficient means to separate hydration phases from unhydrated clinker phases. To this end, for each direction, the $(M, H)$ test results are fitted to the analytical relations (10) and (11) using the minimization procedure (16), with one modification: the minimization procedure is only carried out w.r.t. $(M, H)$ values that yield a packing density smaller than one. $(M, H)$ values yielding a higher indentation response are considered unhydrated clinker phases, which are well-known to exhibit much higher stiffness and strength properties than hydration products. ${ }^{35}$ Figure 4 shows the results of this filter-fitting procedure in terms of $(M, H)$ packing density scaling relations, and Table $\mathrm{V}$ provides the $\mathrm{C}-\mathrm{S}-\mathrm{H}$ particle properties together with relative mean errors and standard deviations of error of the fit. An additional information that is provided by this filtering-fitting method is an estimate of the hydration degree, obtained by dividing the number of tests
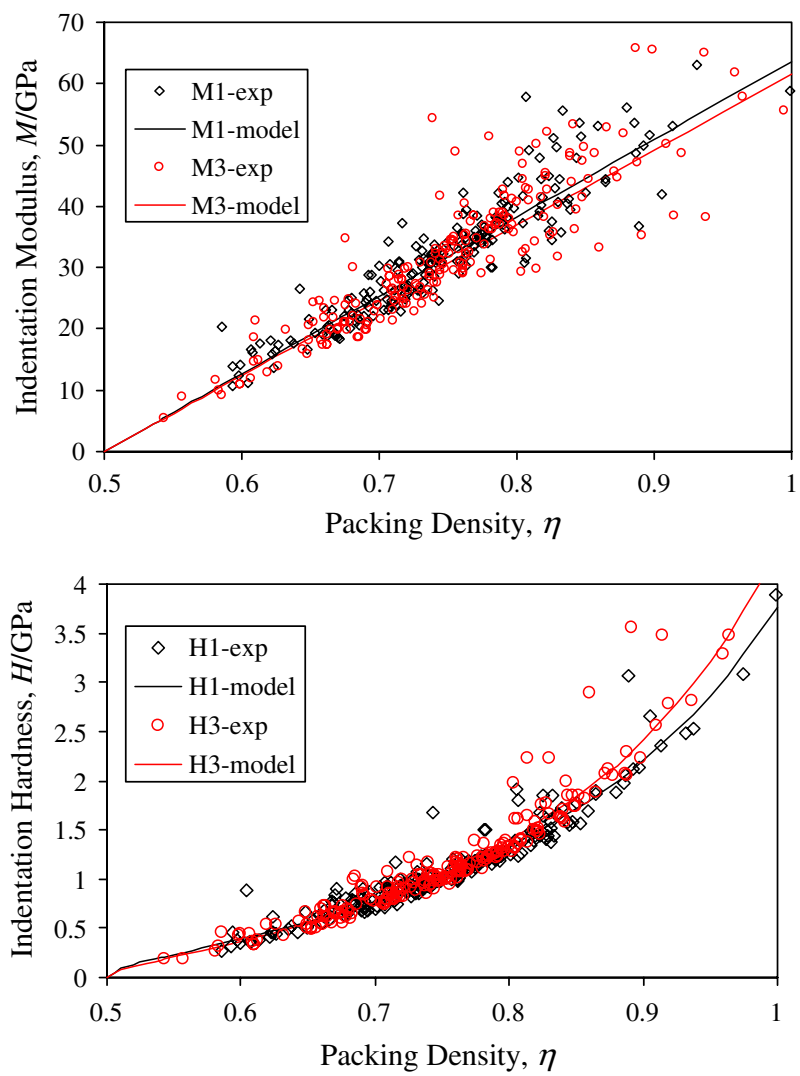

Fig. 4. Isotropy of C-S-H particle properties: Packing density scaling relations of indentation modulus $M$ (top) and indentation hardness $H$ (bottom) obtained from nanoindentation tests on $w / c=0.3$ ordinary portland cement paste, in two orthogonal directions: $M_{1}=M\left(x_{1}\right)$ and $H_{1}=H\left(x_{1}\right)$ are indentation data obtained from surface indentation normal to the cylinder axis, and $M_{3}=M\left(x_{3}\right)$ and $H_{3}=H\left(x_{3}\right)$ are indentation data obtained from indentation parallel to the cylinder axis. identified as hydration products by the total amount of tests. Besides the $x_{1}$ and $x_{3}$ data analyzed separately, Table $\mathrm{V}$ also summarizes the results of a fitting that considers all tests, i.e., both $x_{1}$ and $x_{3}$ data. All results provide a first element of answer to the posed question: the $(M, H)$-packing density scaling of orthogonal directions is almost the same (Fig. 4). As a result, the $\mathrm{C}-\mathrm{S}-\mathrm{H}$ particle exhibits almost the same properties in both indentation directions (Table V); hence, the particle is isotropic, at a scale at least one order of magnitude smaller than the indentation depth, i.e., at the $10-\mathrm{nm}$ scale.

(C) Isotropic $C-S-H$ Packing Density Distributions: The deconvolution of $(M, H, \eta)$ according to (17) completes the picture: Figs. 5(a) and (b) display the experimental cumulative distribution of $M$ and $H$, showing literally the same CDFs for indentation modulus and hardness in orthogonal directions. Table VI summarizes the results of the deconvolution, on the one side separately for the two directions, $x_{1}$ and $x_{3}$, and on the other for all the indentation data of indentation in orthogonal directions. In this deconvolution, three phases sufficed to deconvolute the data. The mean phase properties $(M, H, \eta)$ for the three phases show a fair amount of consistency, and affirm the isotropy of the $\mathrm{C}-\mathrm{S}-\mathrm{H}$ particle packing in hydrated cement pastes. They are also in perfect agreement with the deconvoluted phase properties of the $w / c=0.5$ white cement paste (Table I), showing that all that differs between the hydrated cement paste of different mix proportions are the volume proportions of the following characteristic $\mathrm{C}-\mathrm{S}-\mathrm{H}$ phases:

(1) The lowest packing of $\mathrm{C}-\mathrm{S}-\mathrm{H}$ particle that might be detected in cement pastes is a $\mathrm{C}-\mathrm{S}-\mathrm{H}$ packing close to the random-loose packing fraction of uniform spheres of $0.555 \pm 0.005$, which corresponds to a sphere packing at its rigidity-percolation threshold. ${ }^{25}$ A looser packing, below the percolation threshold $\eta \leq 0.5$, associated with a zero composite strength and stiffness may well exist, but it is impossible to mechanically indent a material with zero stiffness. We found such a random-loose packed phase in a very small volume fraction $(5 \%)$ in the $w / c=0.5$ white cement paste, while it is absent in the $w / c=0.3$ cement paste.

(2) The LD C-S-H phase has a characteristic mean packing density of $64 \%-65 \%$, which comes remarkably close to the random limit packing density of spheres of $\eta \approx 0.64$, which corresponds to the maximum packing density in the random close-packed limit (known as RCP). ${ }^{36, *}$ The LD C-S-H phase is the dominating hydration phase in high water:cement ratio materials, and can be strongly reduced by reducing the water: cement ratio.

(3) The HD C-S-H phase has a characteristic mean packing density of $74 \%-75 \%$, which comes remarkably close to the densest possible spherical packing in three-dimensions of $\eta=\pi / \sqrt{18} \approx 0.74$, which is the ordered face-centered cubic (fcc) or hexagonal close-packed (hcp) packing. ${ }^{37}$ One could argue that this limit packing could also be achieved by monosized ellipsoids, ${ }^{38}$ yet an ellipsoidal packing is associated with a percolation threshold below $0.5,{ }^{26,27}$ which is not consistent with

\footnotetext{
${ }^{\ddagger}$ More recent concepts refer to the RCP as the maximally random jammed state (MRJ), corresponding to the least ordered among all jammed packings, which has been shown to have a density of $63.7 \%$, and which is very close to the traditional definition of the random close-packed limit ${ }^{38}$.
} 
Table V. C-S-H Particle Properties Obtained by Fitting the Dominant Hydration Phase Properties $M$ and $H$ of the $w / c=0.3$ Cement Paste to the Stiffness- and Hardness-Packing Density Relations

\begin{tabular}{|c|c|c|c|}
\hline $\mathrm{C}-\mathrm{S}-\mathrm{H}$ particle properties & $x_{1}^{\dagger}$ & $x_{3}^{\dagger}$ & All \\
\hline Stiffness, $m_{s}(\mathrm{GPa})$ & 63.6 & 61.4 & 62.1 \\
\hline Poisson's ratio, ${ }^{\ddagger} v_{s}$ & 0.2 & 0.2 & 0.2 \\
\hline Cohesion, ${ }^{\S} c_{s}(\mathrm{GPa})$ & 0.501 & 0.434 & 0.463 \\
\hline Friction coefficient, ${ }^{\S} \alpha_{s}$ & 0.141 & 0.217 & 0.179 \\
\hline Friction angle, ${ }^{\top} \varphi$ (deg.) & 8.15 & 12.66 & 10.37 \\
\hline Hydration degree $/ 100$ & 81 & 83 & 83 \\
\hline Mean relative error on $M, \bar{e}_{M} / 100$ & -1.6 & -2.4 & -1.9 \\
\hline Mean relative error on $H, \bar{e}_{H} / 100$ & -1.1 & -1.1 & -1.2 \\
\hline Standard deviation of relative error on $M, \overline{\bar{e}}_{M} / 100$ & 12.4 & 14.5 & 13.5 \\
\hline Standard deviation of relative error on $H, \bar{e}_{H} / 100$ & 10.3 & 10.8 & 10.8 \\
\hline
\end{tabular}

$x_{1}$ stands for indentation on a surface oriented normal to the direction of the cylinder axis, and $x_{3}$ for indentation on surface oriented by the cylinder axis. ${ }^{\ddagger}$ In the fitting, the Poisson's ratio was set to $v_{s}=0.2$. ${ }^{\S}$ Cohesion and friction coefficient are to be understood in the sense of the Drucker-Prager strength model. ${ }^{\top}$ Friction angle is to be understood in the sense of the Coulomb material model, i.e. angle of repose. C-S-H, calcium-silicate-hydrate.

the statistical indentation results. The $\mathrm{HD} \mathrm{C}-\mathrm{S}-\mathrm{H}$ phase is a minor phase in high water:cement materials, and becomes dominant as the water:cement ratio is lowered.

(4) An ultra-HD C-S-H phase (UHD) seems also achievable in cement-based materials, having a characteristic mean packing density of $85 \%-86 \%$. Clearly, such a high packing density cannot be achieved with mono-sized spheres, but hints to packing of particles of different size. Indeed, the found packing density comes remarkably close to a two-scale random limit packing of $\eta=2 \times 0.64-0.64^{2}=0.87$.

It is important to keep in mind that these values are mean packing densities, and that $\mathrm{C}-\mathrm{S}-\mathrm{H}$ particles have some distribution of packing densities. This is illustrated in Figs. 5(c) and (d), showing the experimental CDFs for the two directions (Fig. 5(c)) and the resulting deconvoluted PDFs (Fig. 5(d)) for
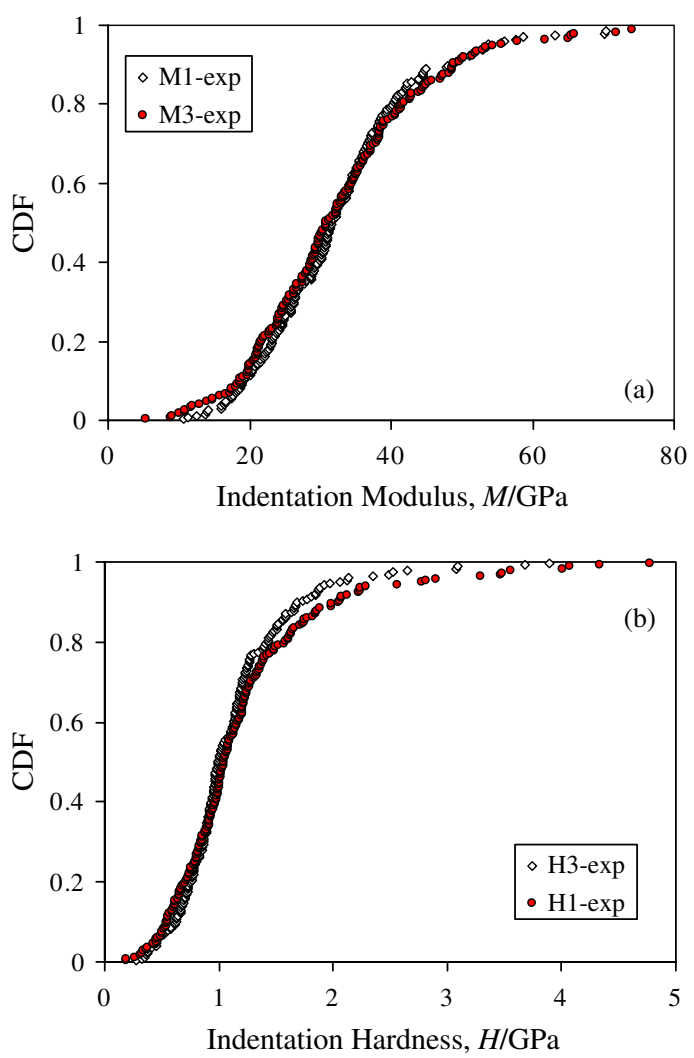

the $w / c=0.3$ cement paste. The CDFs are remarkable as they provide clear evidence of an isotropic distribution of the packing density in orthogonal directions. In its turn, the PDFs show the actual packing density distributions that are obtained on the basis of the employed nanoindentation technique.

Finally, it becomes possible to evaluate the total porosity of the material from

$$
\phi_{0}=\sum_{j=1}^{N} f_{j}\left(1-\eta_{j}\right)
$$

Application of (20) to the $w / c=0.5$ white cement paste yields $\phi_{0}=0.314$, and $\phi_{0}=0.248$ for the $w / c=0.3$ cement paste. Given
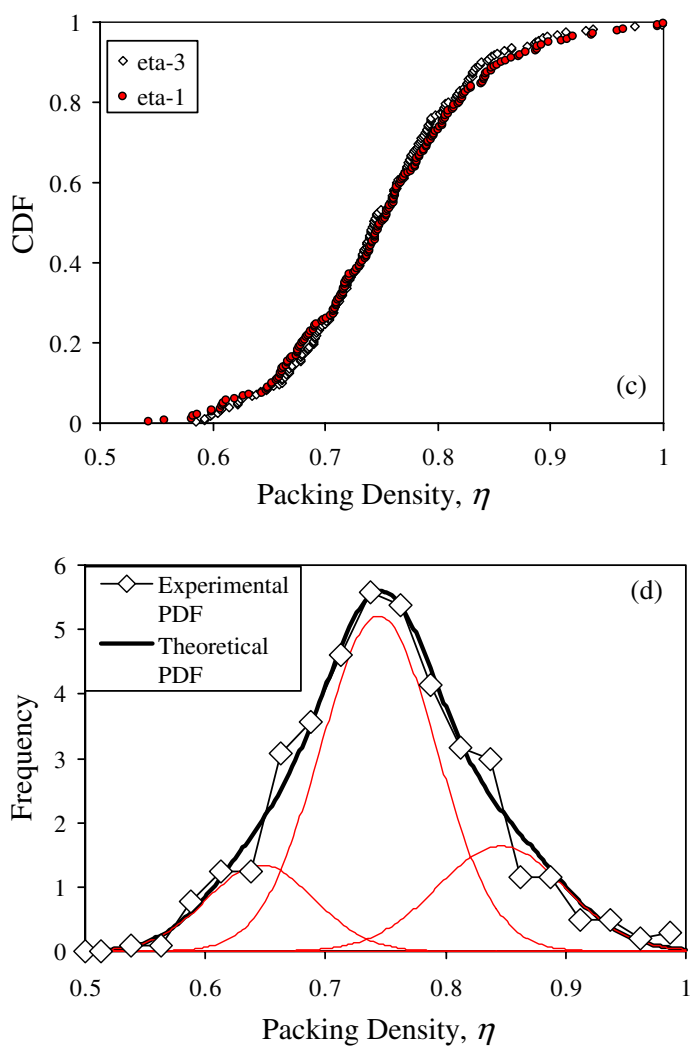

Fig. 5. Statistical Indentation Analysis of C-S-H in hydrated $w / c=0.3 \mathrm{OPC}$ (ordinary Portland cement): The cumulative distribution functions (CDF) of (a) indentation modulus $M$, (b) indentation hardness $H$, and (c) packing density $\eta$ in two orthogonal directions $\left(x_{1}\right.$ is the radial direction, $x_{3}$ is the axial direction) provide evidence of the isotropy of hydration products in cement paste. (d) probability density functions (PDF), derived from the CDF of all tests. 
Table VI. Deconvolution Results of 300 Indentation Tests of a $w / c=0.3$ Ordinary Portland Cement (OPC) in Orthogonal Directions

\begin{tabular}{|c|c|c|c|c|c|c|c|c|}
\hline \multirow[b]{2}{*}{ Direction } & \multirow[b]{2}{*}{ Phase $j$} & \multicolumn{2}{|c|}{$M(\mathrm{GPa})$} & \multicolumn{2}{|c|}{$H(\mathrm{GPa})$} & \multirow{2}{*}{$\begin{array}{l}\text { Surface/volume fraction } \\
f_{j}\end{array}$} & \multicolumn{2}{|c|}{ Packing density } \\
\hline & & $\mu_{j}^{M}$ & $s_{j}^{M}$ & $M(\mathrm{GPa})$ & $s_{j}^{H}$ & & $\mu_{j}^{\eta}$ & $s_{j}^{\eta}$ \\
\hline \multirow[t]{3}{*}{$x_{1}$} & 1 & 18.59 & 3.06 & 0.57 & 0.19 & 0.12 & 0.645 & 0.047 \\
\hline & 2 & 31.27 & 6.85 & 1.00 & 0.24 & 0.70 & 0.742 & 0.051 \\
\hline & 3 & 48.41 & 10.29 & 1.81 & 0.29 & 0.18 & 0.846 & 0.053 \\
\hline \multirow[t]{3}{*}{$x_{2}$} & 1 & 19.70 & 5.05 & 0.61 & 0.22 & 0.23 & 0.656 & 0.044 \\
\hline & 2 & 31.34 & 6.58 & 1.07 & 0.24 & 0.53 & 0.750 & 0.044 \\
\hline & 3 & 47.13 & 9.21 & 1.95 & 0.64 & 0.24 & 0.848 & 0.055 \\
\hline \multirow[t]{3}{*}{ All } & 1 & 19.03 & 4.75 & 0.57 & 0.20 & 0.15 & 0.646 & 0.045 \\
\hline & 2 & 30.75 & 6.97 & 1.02 & 0.25 & 0.63 & 0.744 & 0.048 \\
\hline & 3 & 46.99 & 9.27 & 1.85 & 0.59 & 0.22 & 0.846 & 0.053 \\
\hline
\end{tabular}

$M$, indentation modulus; $H$, indentation hardness.

the difficulty of estimating the porosity of cementitious materials with classical methods, ${ }^{39}$ the statistical indentation technique provides a new nonintrusive way of determining the porosity of nanogranular materials from their nanomechanical contact response.

\section{(2) Intrinsic Isotropy: Structural Anisotropy of Anorganic Bone}

The second application deals with bone, or more precisely with the apatite mineral phase that makes up the ultrastructure of bone, and which imparts to the tissue its mechanical properties and serves as an important depot for calcium and certain polypeptides. Similar to the cement science community, there has been an ongoing debate in the biomechanics community as to whether the macroscopic anisotropy of bone results from the minerals or the collagen proteins that organize in many bones into elementary collagen-mineral units known as fibrils. ${ }^{40}$ The traditional school of thought attributes the anisotropy to the minerals, considering a collagen matrix template reinforced by oriented hydroxyapatite minerals. ${ }^{41,42}$ In more recent years, evidence was brought to light that the ultrastructure of bone is composed of rather randomly oriented apatite particles of different shapes, reinforced by cross-linked collagens. ${ }^{30,43}$ The focus of this section is to re-evaluate the question using the proposed statistical nanoindentation technique.

(A) Materials: Investigation of the collagen-mineral complex in bone is challenged by the high density of the mineral phase that generally obscures the individual crystallite morphology and the relationship of the crystallites to the collagen template. ${ }^{44}$ For this reason, we focus this investigation of the morphology and organization of apatite crystallites on adult bovine cortical bone from which most of the organic material was removed (viz., anorganic bone). This was achieved by a $400^{\circ} \mathrm{C}$ heat treatment for 5 days until no weight change was recorded. ${ }^{45,7}$ The sample preparation, characterization, and experimental protocols of the tested adult bovine cortical bone have been reported previously. ${ }^{46}$ The mineral content of the tested bone specimen was found to be 58 weight percent (wt \%).

(B) Indentation Test Parameters and Results: Denoting by $x_{3}$ the long bone axis, nanoindentation tests were carried out on two orthogonal surfaces: one cut perpendicular to the long bone axis (indentation direction $x_{3}$ ), the other parallel to the long bone axis (indentation direction $x_{1}$ ). In both directions, some 170 force-driven indentation tests were carried out to a maximum load of $P=99 \pm 2 \mu N$. The resulting average maximum indentation depth was found to be greater in the $x_{1}$ direction $(h=45 \pm 11 \mathrm{~nm})$ than in the $x_{3}$ direction $(h=34 \pm 10$ $\mathrm{nm}$ ), providing some first evidence of some anisotropy of the tested microstructure.

The statistical indentation technique provides the following useful information:
(1) Minimization of the $(M, H)$ packing density scaling relations shows an almost isotropic elastic particle behavior for both directions: the scaling with the packing density of the elastic stiffness is almost identical (Fig. 6, top), yielding almost the same asymptotic particle stiffness, $m_{s}\left(x_{1}\right) \approx m_{s}\left(x_{3}\right)=124$ $\mathrm{GPa}$; see Table VII. This asymptotic stiffness matches favorably well with the known elastic constants of hydroxyapatite in powder form; that is $m_{s}^{\exp }=E_{s} /\left(1-v_{s}^{2}\right)=123 \mathrm{GPa}$ (for a Poisson's ratio $\left.v_{s}=0.27\right){ }^{49} \mathrm{~A}$ second independent verification of our scaling results is obtained by comparing the nanoindentation elasticity results with $10 \mathrm{MHz}$ ultrasonic velocity measurements of isotropic hyperpycnotic tissues like the otic bones of whales ${ }^{48}$ or enamel, ${ }^{47}$ which have a very small collagen volume fraction and virtually no microporosity. Hence, such tissues should primarily depend on the packing density of the hydroxyapatite minerals at all observation scales, from several hundred nanometers to several millimeters or centimeters. ${ }^{50}$ The comparison, which is shown in Fig. 6 (top - data labeled 'UPV'), provides clear evidence that the mineral phase that is present in the tested anorganic bovine bones is identical with the mineral phase in hyperpycnotic tissues, and that all what matters is the apatite packing density.

(2) The same minimization for the two directions separately shows some anisotropic trends regarding the strength behavior (Fig. 6, bottom), which is primarily reflected in a higher friction angle for indentation in the $x_{1}$ direction compared with the $x_{3}$ direction (Table VII). Nevertheless, the difference appears to be small, as a simultaneous fit of all data shows, converging with a similar accuracy to a particle cohesion of $c_{s}=1 \mathrm{GPa}$ and a friction angle of $\varphi=22^{\circ}$. This friction value is fairly consistent with the friction angle reported from angle of repose measurements of deorganified bone powder made of the same material, which was found to be $\varphi=33^{\circ}$ in the ambient state and $\varphi=18^{\circ}$ in vacuum. ${ }^{7}$

These results, therefore, provide clear evidence that apatite crystallites in adult bovine cortical bone are made up of almost isotropic elementary particles, at a scale at least one order of magnitude smaller than the indentation depth, i.e., at the unit digit nanometer scale. Anisotropy, if any, therefore, can only originate at larger scales. In other words, the anisotropy of anorganic bone is not intrinsic, but rather structural.

(C) Anisotropic Packing Density Distributions: The existence of a structural anisotropy in both stiffness and strength can be grasped from the CDFs of $M$ and $H$ in Figs. 7(a) and (b). There is a significant difference in the stiffness and strength distribution in bone's ultrastructure in the axial and radial bone direction, respectively. This structural anisotropy is found to originate from an anisotropic packing density distribution, as shown by the deconvolution results of $(M, H, \eta)$ summarized in Table VIII and illustrated in Figs. 7(c) and (d). In particular, reminding ourselves that the particles are isotropic (see Fig. 6 and Table VII), the structural anisotropy is recognized to be introduced by a denser particle packing in the axial $\left(x_{3}\right)$ direc- 

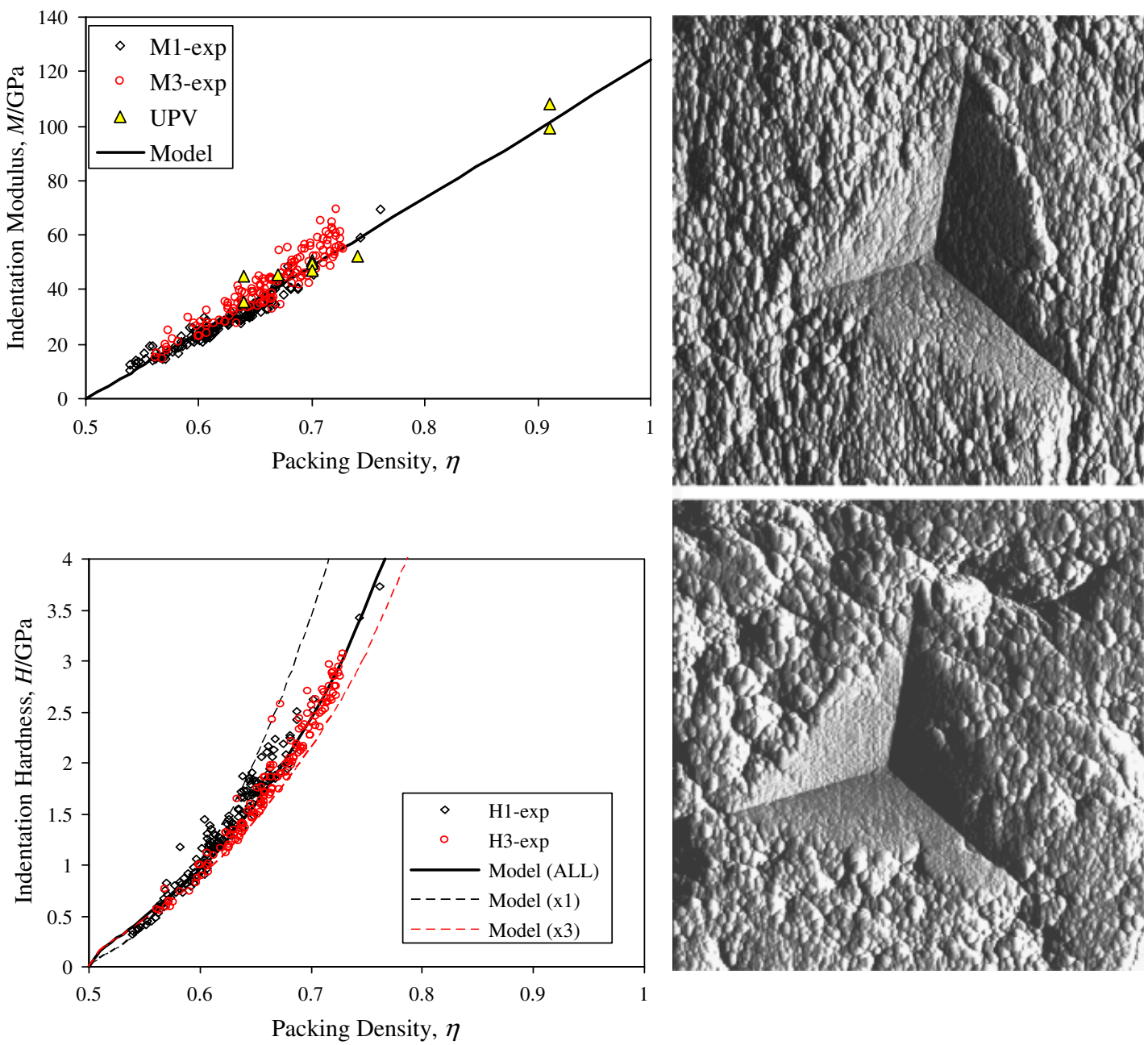

Fig. 6. Statistical Isotropy of apatite mineral particle in anorganic bone: Packing density scaling relations of indentation modulus $M$ (top) and indentation hardness $H$ (bottom) obtained from nanoindentation tests on anorganic bone, in two orthogonal directions: $M_{1}=M\left(x_{1}\right)$ and $H_{1}=H\left(x_{1}\right)$ are indentation data obtained from surface indentation normal to the long bone axis, and $M_{3}=M\left(x_{3}\right)$ and $H_{3}=H\left(x_{3}\right)$ are indentation data obtained from indentation in the long bone axis. The top figure also shows some results from $10 \mathrm{MHz}$ ultrasonic tests (UPV) on isotropic hyperpycnotic tissues like the otic bones of whales or enamel, tested by Lees and Rollins ${ }^{47}$ and Lees et al. ${ }^{48}$ For these tissues, the hydroxyapatite packing density was determined from the wet tissue mass density and the weight fraction of minerals [from the compilation of data in Hellmich and Ulm ${ }^{30}$. The right figures are topographic AFM images of residual imprints from nanoindentation experiments on deorganized bone (maximum load $=7 \mathrm{mN}$, Berkovich probe geometry, $7 \mu \mathrm{m}$ scan size).

tion than in the radial $\left(x_{1}\right)$ direction. The statistical indentation technique, therefore, depicts a radial packing density gradient of the crystallite phase in the deorganified bone material. On the other hand, the total porosity determined from (20) is roughly the same; $\phi_{0}=0.38 \pm 0.04$ for direction $x_{1}$ and $\phi_{0}=0.32 \pm 0.04$ for direction $x_{3}$. The dominating phase in both directions has a characteristic particle packing density of $\eta=0.62-0.66$, that is on the order of the random limit packing density of spheres of $64 \%$. The higher packing density of the secondary phase sensed by nanoindentation in the $x_{3}$ direction comes close to the ordered fcc or hep packing of $74 \%$.

In summary, what differs between $\mathrm{C}-\mathrm{S}-\mathrm{H}$ and anorganic cortical bovine bone is a radial packing density gradient. This packing density gradient, which is most likely related to a privileged osteonic growth direction related to natural growth and remodeling cycles in bone, induces bone's macroscopic anisotropy at a scale much greater than the chosen scale of nanometer-sized contact mechanics.

\section{(3) Intrinsic Particle Anisotropy in Sedimentary Rocks: Shale}

The last application deals with shale, the sealing formation in most hydrocarbon reservoirs. Among hydrated nanocompo- sites, shale is most probably the most complex material. Shale is a multiphase, multiscale, transversely isotropic, and compositionally diverse sedimentary rock that is made of highly compacted clay particles of submicrometer size, nanometric porosity, and silt inclusions. Implementing the materials science paradigm for such materials has been a challenge due to the highly heterogeneous nature of shales, which span roughly nine orders of magnitude, from the tens of nanometer scale of the plate- or sheet-like clay particles with an average thickness-to-diameter aspect ratio of $1 / 20$, to the macroscale of the layered rock composite composed of a porous clay phase and silt-sized quartz inclusions. ${ }^{51}$ The discussion that goes on in the shale acoustics community as to the source of anisotropy of shale is reminiscent of that in the cement science and biomechanics community. Indeed, starting with Kaarsberg's first velocity measurements in $1959,{ }^{52}$ the classical school of thought attributes macroscopic shale anisotropy to the deposition history of the sedimentary rock, demonstrating that anisotropy tends to increase with a decrease in porosity. More recent approaches based on effective medium theory derive shale anisotropy from particle shape ${ }^{53}$ and clay-particle orientation distribution functions, ${ }^{54}$ while assuming the existence of a 'perfect' clay particle that is intrinsically isotropic. First nanoindentation results available for a large array of shales of different geographical and depth origin, and different 
Table VII. Apatite Properties Obtained by Fitting the Anorganic Bone Properties $M$ and $H$ to the Stiffness- and Hardness-Packing Density Scaling Relations

\begin{tabular}{|c|c|c|c|}
\hline Apatite particle properties & $x_{1}^{\dagger}$ & $x_{3}^{\dagger}$ & All \\
\hline Stiffness, $m_{s}(\mathrm{GPa})$ & 124.4 & 124.4 & 124.4 \\
\hline Poisson’s ratio, ${ }^{\ddagger} v_{s}$ & 0.27 & 0.27 & 0.27 \\
\hline Cohesion ${ }^{\S} c_{s}(\mathrm{GPa})$ & 0.90 & 0.96 & 1.01 \\
\hline Friction coefficient, ${ }^{\S} \alpha_{s}$ & 0.534 & 0.340 & 0.373 \\
\hline Friction angle, $\varphi$ (deg.) & 34.18 & 20.29 & 22.49 \\
\hline Mean relative error on $M, \bar{e}_{M} / 100$ & -1.0 & -1.0 & -1.3 \\
\hline Mean relative error on $H, \bar{e}_{H} / 100$ & -0.4 & -0.6 & -0.8 \\
\hline Standard deviation of relative error on $M, \overline{\bar{e}}_{M} / 100$ & 9.7 & 9.5 & 11.2 \\
\hline Standard deviation of relative error on $H, \bar{e}_{H} / 100$ & 6.6 & 7.4 & 9.0 \\
\hline
\end{tabular}

${ }^{\dagger} x_{1}$ stands for indentation normal to the long bone axis, and $x_{3}$ for indentation into long bone axis. ${ }^{\ddagger}$ In the fitting, the Poisson's ratio was set to $v_{s}=0.27$, corresponding to the Poisson's ratio of apatite in powder form. ${ }^{\S}$ Cohesion and friction coefficient are to be understood in the sense of the Drucker-Prager strength model. "Friction angle is to be understood in the sense of the Coulomb material model, i.e. angle of repose.

mineralogy and porosity hint toward an intrinsic elastic anisotropy, ${ }^{5}$ and show some consistent scaling of elasticity and hardness with the clay packing density. ${ }^{6}$ The focus of the application of the proposed statistical indentation technique to shale is to identify the source of shale anisotropy.

(A) Material and Surface Preparation: The shale material is from small core samples preserved in desiccators with salt solutions in conditions near to the natural relative humidity of the material. The shale, which is benchmark shale \#3 of the GeoGenome project, ${ }^{5}$ has a total porosity of $\phi_{0}=0.075-0.076$, a nonclay silt inclusion volume fraction of $f^{\text {inc }}=0.274-0.275$, and a solid clay volume fraction of $f^{c}=0.650$, of which $8.4 \%$ was identified as kaolonite, $50.6 \%$ as illite-smectite, and $6 \%$ as others (chlorite, glauconite, biotite). ${ }^{6}$ Mercury intrusion porosi- metry results show that the porosity is situated solely in the clay phase, translating into a clay porosity $\varphi_{0}=\phi_{0} /\left(1-f^{\text {inc }}\right)=$ $0.103-0.104$, respectively, into a clay packing density $\eta=1-$ $\varphi_{0}=f^{c} /\left(1-f^{\text {inc }}\right)=0.896-0.897$

The samples were cut to expose surfaces parallel $\left(x_{3}\right)$ and perpendicular $\left(x_{1}, x_{2}\right)$ to the isotropy plane (deposition direction) of the material. The surface preparation follows the polishing procedure for heterogeneous materials outlined before. From AFM testing, the RMS roughness obtained with this polishing procedure was 128 and $152 \mathrm{~nm}$ in the $x_{1}$ and the $x_{3}$ direction, respectively. These roughness values are on the order of the characteristic size of clay particles.

(B) Indentation Parameters and Results: Scale separability in indentation testing requires to choose an indentation
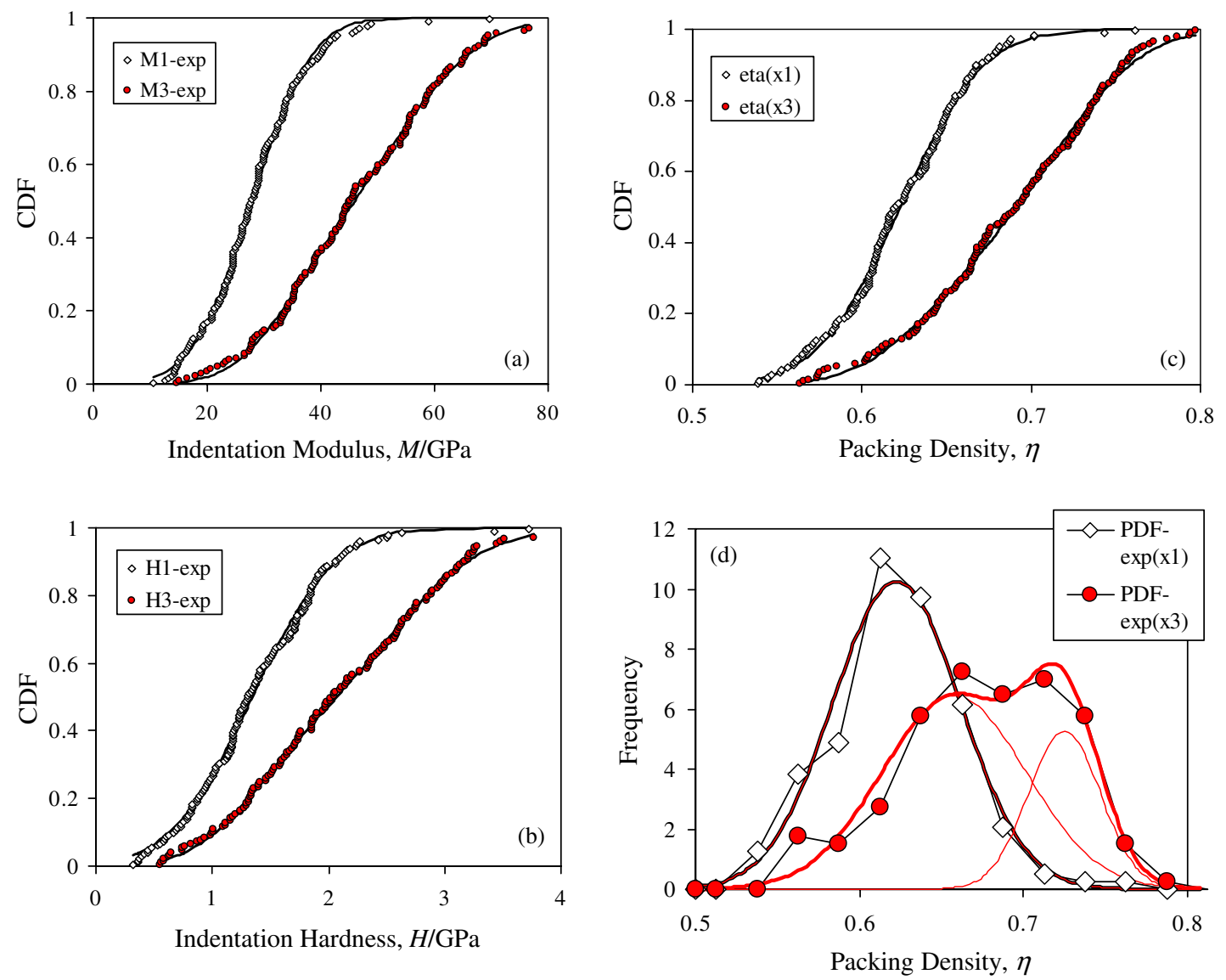

Fig. 7. Statistical indentation analysis of anorganic cortical bone: Cumulative distribution functions (CDF) of (a) indentation modulus $M$, (b) indentation hardness $H$, and (c) packing density $\eta$ in two orthogonal directions: $x_{1}$ is the radial direction (normal to long bone axis), $x_{3}$ is the axial direction (long bone axis). (d) Probability density function (PDF) of the packing density, showing an anisotropic packing density distribution in orthogonal directions. 
Table VIII. Deconvolution Results of Nanoindentation tests on Anorganic Bone, in the Radial $\left(x_{1}\right)$ Direction and in the Axial ( $\left.x_{3}\right)$ Direction

\begin{tabular}{|c|c|c|c|c|c|c|c|c|}
\hline Direction & Phase $j$ & \multicolumn{2}{|c|}{$M(\mathrm{GPa})$} & \multicolumn{2}{|c|}{$H(\mathrm{GPa})$} & $\begin{array}{l}\text { Surface/volume fraction } \\
f_{j}\end{array}$ & \multicolumn{2}{|c|}{ Packing density } \\
\hline$x_{3}$ & 1 & 40.09 & 11.93 & 1.70 & 0.65 & 0.71 & 0.658 & 0.044 \\
\hline & 2 & 61.18 & 9.17 & 2.97 & 0.38 & 0.29 & 0.725 & 0.022 \\
\hline
\end{tabular}

$M$, indentation modulus; $H$, indentation hardness; $\eta$, packing density.

depth sufficiently larger than the elementary size of the clay particles. In addition, in order to apply the continuum indentation model of an infinite half-space, the indentation depth must be larger than the surface roughness, ${ }^{55}$ typically $h>3 R_{q}{ }^{56}$ This is achieved here with a maximum indentation force of $P=4.85 \pm 0.05 \mathrm{mN}$ applied in both directions, which produces, on the specific shale materials tested, an on-average maximum indentation depth that is similar in orthogonal directions: $h=584 \pm 272 \mathrm{~nm}$ in the $x_{1}$ direction, and $h=613 \pm 204 \mathrm{~nm}$ in the $x_{3}$ direction. This average response suggests a fair amount of isotropy in the mechanical response of the indented shale material. However, as we show here below, in the case of shales, this isotropy stems from the morphology, whereas the particle itself is anisotropic.

The CDFs of indentation modulus and indentation hardness shown in Fig. 8 display a statistically anisotropic elasticity behavior $\left(M_{1}>M_{3}\right)$, yet a statistically isotropic hardness beha- vior of the dominating phase sensed by shale nanoindentation, which - at the scale of observation - is the porous clay phase. Further evidence of the hardness isotropy is provided by the AFM photo simulations after a $4.8 \mathrm{mN}$ indent in respectively the $x_{1}$ and $x_{3}$ directions, which are also shown in Fig. 8, and which do not display a significant difference in neither particle morphology nor imprint size.

(C) Isotropic Packing Density Distribution: Anisotropic Particle Properties: At this stage, employing the stiffness and hardness-packing density scaling relations will be useful. Based on the statistical anisotropy of the elasticity of the clay particle, and the statistical isotropy of the hardness distribution, it is natural to assume that the particle stiffness is anisotropic, whereas the strength behavior is isotropic. Hence, the isotropic hardness-packing density scaling relation (11) can still be employed, while the indentation modulus-packing density scaling (10) requires adaptation.
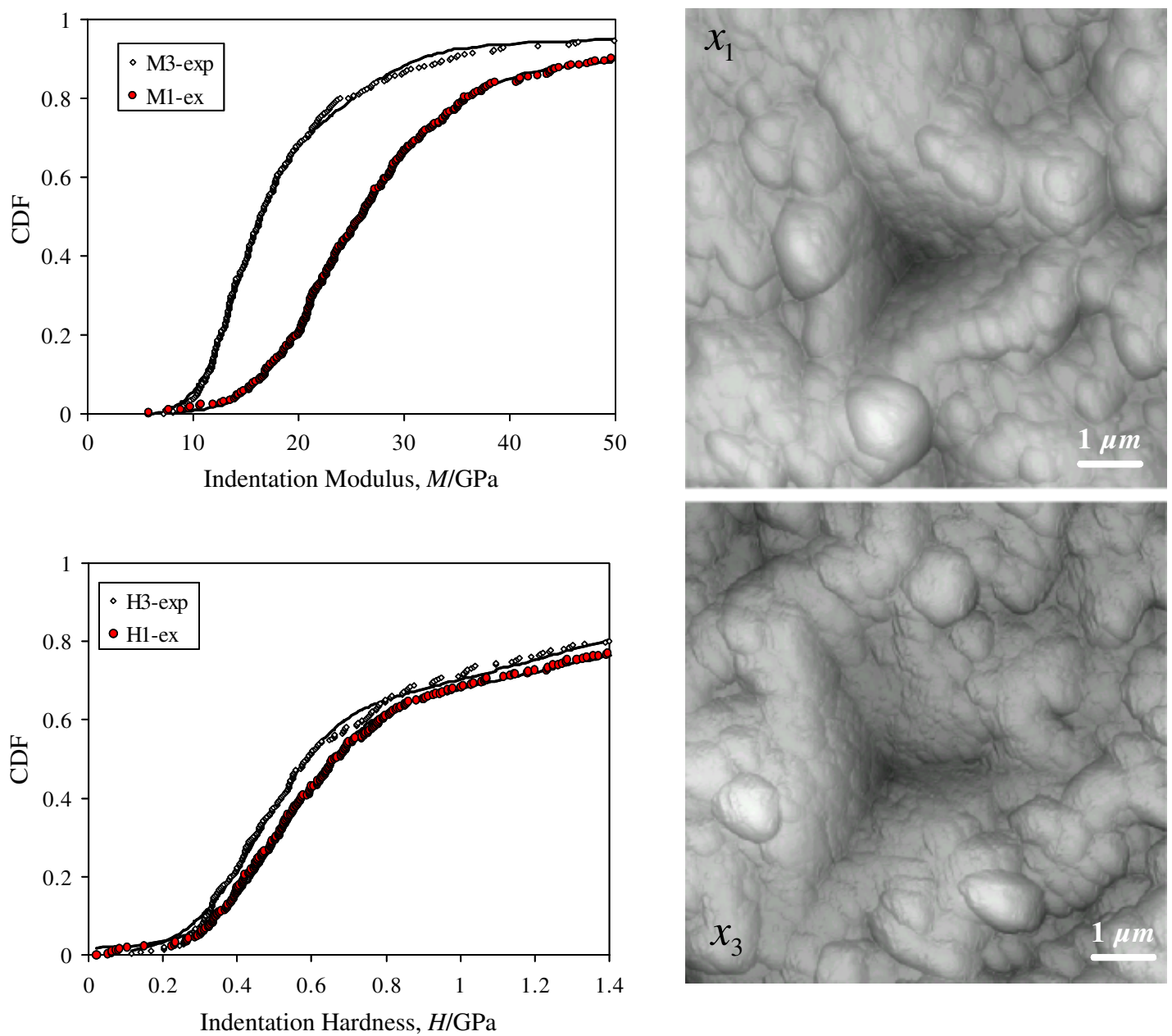

Fig. 8. Evidence of intrinsic elastic anisotropy morphological isotropy of shales: cumulative distribution functions (CDF) of indentation modulus (top left) and indentation hardness (bottom left) in two orthogonal directions: $x_{3}$ is the material symmetry axis corresponding to the deposition direction, and $x_{1}$ lies in the bedding plane. The right figures are topographic AFM images of residual imprints from nanoindentation experiments on shale in orthogonal directions (maximum load $=4.8 \mathrm{mN}$, Berkovich probe geometry, $8 \mu \mathrm{m}$ scan size). 

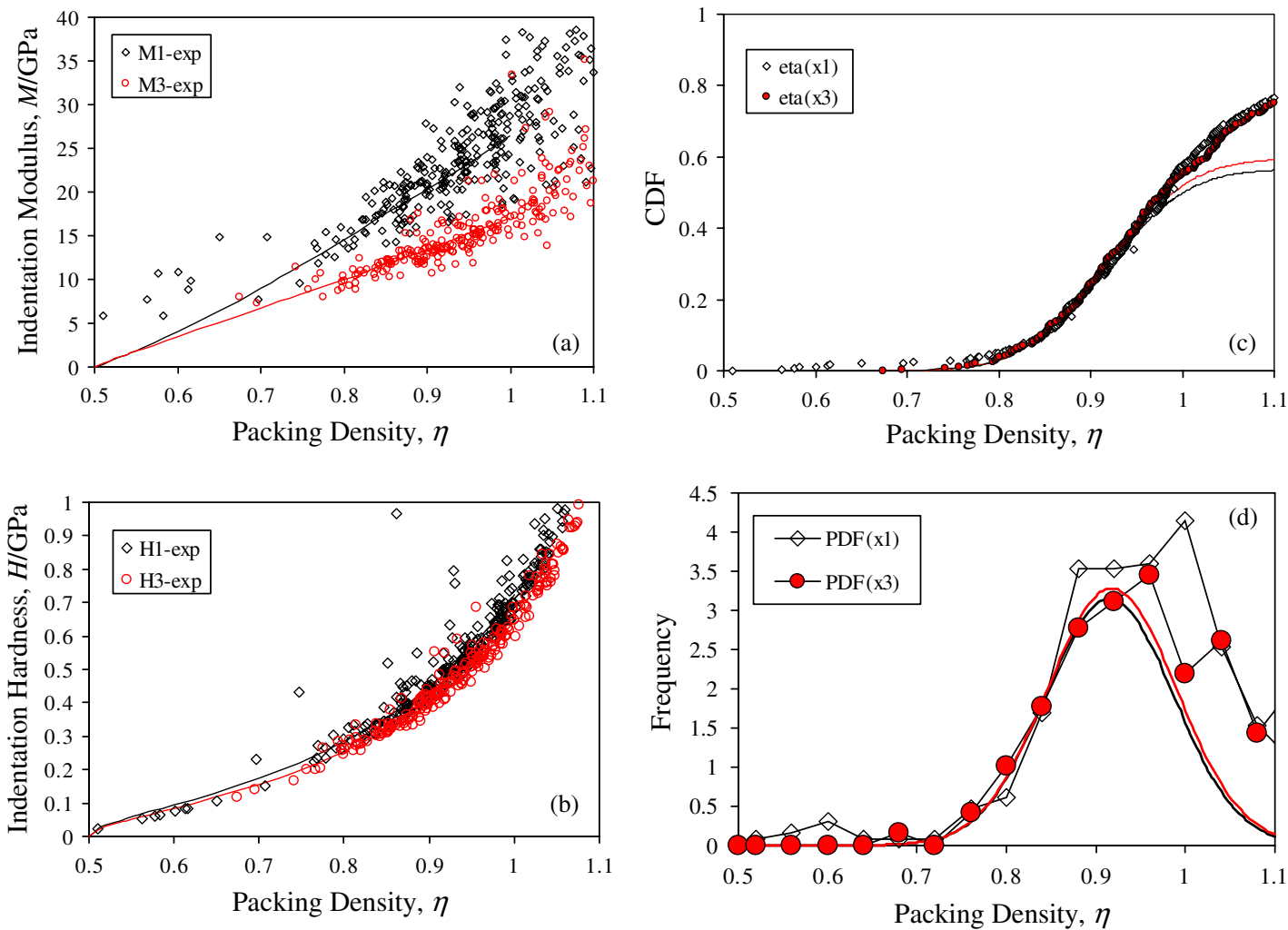

Fig. 9. Intrinsic anisotropy of shale clay elasticity and isotropic packing density distribution: (a) indentation moduli-packing density scaling relations, (b) indentation hardness-packing density scaling relations, (c) cumulative distribution function, and (d) probability density function of clay packing density distribution.

This is achieved here by application of micromechanics to a porous medium composed of an elastically anisotropic solid and porosity: ${ }^{24}$

$$
\mathbb{C}_{\text {hom }}=\mathbb{C}^{s}:\left(\rrbracket-(1-\eta) \overline{\mathbb{A}}^{p}\right)
$$

where $\mathbb{C}^{s}$ is the tensor of clay stiffness values, whereas $\overline{\mathbb{A}}^{p}$ stands for the so-called strain localization tensor. ${ }^{23}$ Given the visible isotropic morphology, it is appropriate to estimate this strain localization tensor by the self-consistent scheme for transversely isotropic particles, for which ${ }^{57}$ :

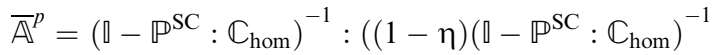

$$
\begin{aligned}
& \left.+\eta\left(\mathbb{\square}+\mathbb{P}^{S C}:\left(\mathbb{C}^{s}-\mathbb{C}_{\text {hom }}\right)\right)^{-1}\right)^{-1}
\end{aligned}
$$

where $\mathbb{P}^{S C}$ is the fourth-order Hill tensor characterizing the interaction between particles. The self-consistent scheme has a long tradition in the application of micromechanics to shales, ${ }^{53}$ most probably due to its unique feature of representing a highly disordered composite response of interacting phases none of which play a specific morphological matrix or inclusion role. ${ }^{58-62}$ This makes the self-consistent scheme most suitable for a porous composite whose solid phase is a granular material. The main difference of our approach with previous approaches is that both the solid phase and the homogeneous material are transversely isotropic, which makes the determination of the Hill tensor $\mathbb{P}^{\mathrm{SC}}$ quite involving. ${ }^{63}$ Fortunately, Hellmich et al. ${ }^{50}$ have recently addressed the problem of spherical transversely isotropic inclusions through exploiting the symmetries involved in the specification of the general ellipsoidal anisotropic case, resulting in an expression of $\mathbb{P}^{\mathrm{SC}}$ that can be evaluated by the numerical solution of some single-variable integrals (for details, see, Hellmich and colleagues $\left.{ }^{50,57}\right)$. Finally, the in situ stiffness of solid clay particles $\mathbb{C}^{s}=\lim _{\eta=1}\left(\mathbb{C}_{\text {hom }}\right)$ has been recently determined from backanalysis of velocity measurements of many shale materials ${ }^{57}: C_{11}^{s}=44.9 \mathrm{GPa}, C_{12}^{s}=21.7 \mathrm{GPa}$, $C_{13}^{s}=18.1 \mathrm{GPa}, C_{33}^{s}=24.2 \mathrm{GPa}$, and $C_{44}^{s}=3.7 \mathrm{Gpa}$. On this basis, use of $\mathbb{C}_{\text {hom }}$ (Eq. (21)) in the indentation modulus relations (4) and (5) yields packing density scaling relation of the indentation moduli in the form

$$
\begin{aligned}
& \frac{M_{i}}{m_{i}^{s}}=\Pi_{M_{i}} \\
& \left(\frac{C_{11}^{s}}{C_{33}^{s}}=1.9, \frac{C_{11}^{s}-C_{12}^{s}}{2 C_{44}^{s}}=3.1, \frac{C_{33}^{s}-C_{13}^{s}}{2 C_{44}^{s}}=0.8, \frac{C_{12}^{s}}{C_{13}^{s}}=1.2, \eta, \eta_{0}=1 / 2\right)
\end{aligned}
$$

where $m_{i}^{s}(i=1,3)$ are the solid indentation moduli in the orthogonal directions $x_{1}$ and $x_{3}$. For practical application in the minimization problem (16), we fit the dimensionless expressions $\Pi_{M_{i}}$ in power functions of the packing density

$$
\begin{aligned}
\Pi_{M_{3}}= & -2.43\left\langle\eta-\eta_{0}\right\rangle^{4}+3.14\left\langle\eta-\eta_{0}\right\rangle^{3} \\
& -1.15\left\langle\eta-\eta_{0}\right\rangle^{2}+2.10\left\langle\eta-\eta_{0}\right\rangle \\
\Pi_{M_{1}}= & 3.54\left\langle\eta-\eta_{0}\right\rangle^{4}-5.05\left\langle\eta-\eta_{0}\right\rangle^{3} \\
& +3.09\left\langle\eta-\eta_{0}\right\rangle^{2}+1.28\left\langle\eta-\eta_{0}\right\rangle
\end{aligned}
$$

where $\langle x\rangle=(x+|x|) / 2$. Despite the fact that $\Pi_{M_{3}} \neq \Pi_{M_{1}}$, one recognizes that those functions do not differ much from a straight line relation $\langle 2 \eta-1\rangle$ within the admissible interval $\eta_{0}=1 / 2 \leq \eta \leq 1$; and that the anisotropy of the elementary particle is essentially contained in the solid indentation moduli, $m_{3}^{s} \neq m_{1}^{s}$.

Application of the packing density relations (anisotropic elasticity and isotropic strength) to the indentation results of orthogonal directions yields the results displayed in Fig. 9. In particular 
(1) The indentation moduli-packing density plot (Fig. 9(a)) shows that the anisotropy increases with increasing packing density, from the percolation threshold $\eta_{0}=1 / 2$ to the quasisolid state $\eta=1$, beyond which the data cannot be fitted to the scaling relation. Indeed, at the chosen scale of indentation, shale nanoindentation senses the mechanical response of three phases: the dominating porous clay phase $(60 \%)$, the composite response of the clay phase with silt inclusions, and the pure silt response. The (elastic) anisotropy is maximum for the load bearing clay phase, and significantly decreases for both the composite phase and the silt phase. The asymptotic values of the particle stiffness are $m_{3}^{s}=\lim _{\eta=1} M\left(x_{3}\right)=17.0 \mathrm{GPa}$ and $m_{1}^{s}=\lim _{\eta=1} M(x 1)=26.4 \mathrm{GPa}$, and are in very good agreement with the clay stiffness values one determines from using the before-mentioned $\mathbb{C}^{s}$ values in Eqs. (4) and (5): $M_{3} \times$ $\left(\mathbb{C}^{S}\right)=14.8 \mathrm{GPa}$ and $M_{1}\left(\mathbb{C}^{s}\right)=26.3 \mathrm{GPa}$.

(2) In contrast, the indentation hardness-packing density plot (Fig. 9(b)) shows almost the same hardness scaling for indentation in orthogonal directions. This is expected given the statistical isotropy of the hardness captured by the CDF (Fig. 8). The particle cohesion is almost the same in both directions, namely $c_{s}\left(x_{3}\right)=0.130 \mathrm{GPa}$ and $c_{s}\left(x_{1}\right)=0.145 \mathrm{GPa}$. Furthermore, for both directions, the friction is found to be zero.

(3) The CDF and the PDF of the clay packing density shown in Figs. 9(c) and (d) complete the picture: The packing density distribution of the load bearing clay phase is almost identical in orthogonal directions, hinting towards an isotropic morphology sensed by nanoindentation. The mean packing density of the load bearing clay phase, obtained from a deconvolution, is the same in orthogonal directions $\eta\left(x_{3}\right)=\eta\left(x_{3}\right)=0.92 \pm 0.07$; and it is in fairly good agreement with the clay packing density determined from mineralogy and porosity data, $\eta=0.90$.

In summary, in contrast to bone (see Figs. 6 and 7), the load bearing clay phase in shale (see Figs. 8 and 9) exhibits an intrinsic elastic anisotropy of the elementary particle. Yet, the packing of those particles, and thus the particle morphology, is isotropic. In other words, despite its deposition history, the clay particle aspect ratio is of secondary importance for the nanomechanical response of shales.

\section{Conclusion}

The overall picture which thus emerges is that hydrated nanocomposites are nanogranular materials, whose nanomechanical behavior is driven by the packing of their elementary particles. The packing of particles has been a focus of intensive research in the granular physics community. ${ }^{25-27,36-38}$ The statistical nanoindentation technique allows one to quantitatively assess in situ the nanogranular state of hydrated nanocomposites and identify in situ mineral properties and intrinsic and structural sources of anisotropy:

(1) The elementary particles present in $\mathrm{C}-\mathrm{S}-\mathrm{H}$, bone, and shale all possess an isotropic strength behavior. We attribute this strength to cohesive bonds that are activated at particle-toparticle contact surfaces, which are expected to be sufficiently smaller than the mineral cohesion itself. The isotropy of the strength behavior hints toward a random orientation of the contact surfaces. Further evidence of this particle-to-particle nanocontact is provided by the nonnegligible friction we find for $\mathrm{C}-\mathrm{S}-\mathrm{H}$ and apatite, which is most likely due to particle-toparticle interlocking. In contrast, the absence of friction in deposited clay is no doubt indicative of a 'smooth' deposition and compaction history, which minimizes particle interlocking while maximizing the packing density according to the clay size distribution.

(2) Particles transmit forces over randomly oriented contact surfaces, activating the intrinsic elasticity of the nanoparticle. In the case of $\mathrm{C}-\mathrm{S}-\mathrm{H}$ and apatite, this intrinsic elasticity is found to be isotropic while it turned out to be anisotropic in the case of the load bearing clay phase in shale. The isotropy of $\mathrm{C}-\mathrm{S}-\mathrm{H}$ and apatite elasticity may well be due to a random orientation of the particles as a result of a random precipitation and growth process of particles. Indeed, during the hydration reactions, nanoparticles precipitate quasi randomly in a calcium oversaturated pore solution (water in the case of $\mathrm{C}-\mathrm{S}-\mathrm{H}$, bone marrow in the case of apatite). These precipitating nanoparticles grow and percolate beyond a packing density of $50 \%$. As the hydration proceeds, the nanogranular units touch each other generating random-oriented contact surfaces, independent of the mineral orientation. In contrast, the anisotropy of the clay elasticity can be attributed to the deposition process of the sedimentary clays, which induces privileged orientation of the mineral, and which gives rise to an anisotropic particle deposition stiffness.

(3) The elementary particles present in cement paste, bone, and shale all possess an isotropic mechanical morphology. By isotropic morphology, we mean one in which the particle shape and aspect ratio do not affect significantly the nanomechanical response. This isotropic morphology is reminiscent of a random orientation of the particle-to-particle contact surfaces over which forces are transmitted. This randomness prevails even in the case of particles having a visible shape as it is the case of clay particles in shales. Such an isotropic morphology is indicative of a percolation threshold of $\eta_{0}=1 / 2$ as recognized by the polycrystal, or selfconsistent model of micromechanics, which turns out to be most suitable for the hydrated nanocomposites.

\section{Acknowledgments}

The MIT Department of Materials Science and Engineering Nanomechanical Testing Facility. The content does not necessarily reflect the position of the funding companies and institutions, and no official endorsement should be inferred.

\section{References}

${ }^{1}$ G. Constantinides, F.-J. Ulm, and K. Van Vliet, "On the Use of Nanoindentation for Cementitious Materials," Mater. Struct., 36 [257] 191-6 (2003).

${ }^{2}$ G. Constantinides and F.-J. Ulm, "The Effect of Two Types of C-S-H on the Elasticity of Cement-Based Materials: Results from Nanoindentation and Micromechanical Modeling," Cement Concrete Res., 34 [1] 67-80 (2004).

${ }^{3}$ G. Constantinides and F.-J. Ulm, "The Nanogranular Nature of C-S-H," J. Mech. Phys. Solids, 55 [1] 64-90 (2007).

${ }^{4}$ M. J. DeJong and F.-J. Ulm, "The Nanogranular Behavior of C-S-H at Elevated Temperatures (up to 700 degrees C)," Cement Concrete Res., 37 [1] 1-12 (2007).

${ }^{5}$ F.-J. Ulm and Y. Abousleiman, "The Nanogranular Nature of Shale," Acta Geotech., 1 [2] 77-88 (2006).

${ }^{6}$ C. Bobko and F.-J. Ulm, "The Nanomechanical Morphology of Shale," Mech. Mater. (in review) (2007).

${ }^{7}$ K. Tai, F.-J. Ulm, and C. Ortiz, "Nanogranular Origins of the Strength of Bone," Nano Lett., 6 [11] 2520-5 (2006).

${ }^{8}$ W. C. Oliver and G. M. Pharr, "Measurement of Hardness and Elastic Modulus by Instrumented Indentation: Advances in Understanding and Refinements to Methodology," J. Mater. Res., 19 [1] 3-20 (2004).

${ }^{9}$ Y. T. Cheng and C. M. Cheng, "Scaling, Dimensional Analysis, and Indentation Measurements," Mater. Sci. Eng. R-Rep., 44 [4-5] 91-149 (2004).

${ }^{10}$ A. Perriot and E. Barthel, "Elastic Contact to a Coated Half-Space: Effective Elastic Modulus and Real Penetration," J. Mater. Res., 19 [2] 600-8 (2004).

${ }^{11}$ G. Constantinides, K. S. R. Chandran, F.-J. Ulm, and K. Van Vliet, "Grid Indentation Analysis of Composite Microstructure and Mechanics: Principles and Validation," Mater. Sci. Eng. A-Struct. Mater. Properties Microstruct. Process., 430 [1-2] 189-202 (2006)

${ }^{12}$ S. Cariou, F.-J. Ulm, and L. Dormieux. Hardness-packing density scaling relationships for cohesive-frictional porous materials. J. Mech. Phys. Solids, in press (2007)

${ }^{13}$ L. A. Galin, Contact Problems in Theory of Elasticity. Translated by H. Moss. Edited by I. N. Sneddon. North Carolina State College, Raleigh, 1961.

${ }^{14}$ I. N. Sneddon, "The Relation Between Load and Penetration in the AxiSymmetric Boussinesq Problem for a Punch of Arbitrary Profile," Int. J. Eng. SC., 3, 47-57 (1965).

${ }^{15}$ J. R. Willis, "Herztian Contact of Anisotropic Bodies," J. Mech. Phys. Solids, 14, 163-76 (1966)

${ }^{16}$ J. J. Vlassak and W. D. Nix, "Indentation Modulus of Elastically Anisotropic Half Spaces," Philos. Magn. A, 67 [5] 1045-56 (1993).

${ }^{17}$ J. J. Vlassak and W. D. Nix, "Measuring the Elastic Properties of Anisotropic Materials by Means of Indentation Experiments," J. Mech. Phys. Solids, 42 [8] 1223-45 (1994).

${ }^{18}$ J. G. Swadener and G. M. Pharr, "Indentation Modulus of Elastically Anisotropic Half-Spaces by Cones and Parabolae of Revolution," Philos. Magn. $A, \mathbf{8 1}, 447-66$ (2001). 
${ }^{19}$ J. J. Vlassak, M. Ciavarella, J. R. Barber, and X. Wang, "The Indentation Modulus of Elastically Anisotropic Materials for Indenters of Arbitrary Shape," J. Mech. Phys. Solids, 51, 1701-2 (2003).

${ }^{20}$ A. Delafargue and F.-J. Ulm, "Explicit Approximations of the Indentation Modulus of Elastically Orthotropic Solids for Conical Indenters," Int. J. Solids Struct., 41 [26] 7351-60 (2004).

${ }^{21}$ H. M. Jennings, J. J. Thomas, J. S. Gevrenov, G. Constantinides, and F.-J. Ulm, "A Multi-Technique Investigation of the Nanoporosity of Cement Paste,' Cement Concrete Res., 37 [3] 329-36 (2007)

${ }^{22} \mathrm{H}$. M. Jennings, "Colloid Model of C-S-H and Implications to the Problem of Creep and Shrinkage," Mater. Struct., 37 [265] 59-70 (2004).

${ }^{23}$ A. Zaoui, "Continuum Micromechanics: Survey," J. Eng. Mechan.-ASCE, 128 [8] 808-16 (2002)

${ }^{24}$ L. Dormieux, D. Kondo, and F.-J. Ulm, Microporomechanics. John Wiley \& Sons, Chichester, UK, 2006.

${ }^{25}$ G. Y. Onoda and E. G. Liniger, "Random Loose Packings of Uniform Spheres and the Dilatancy Onset," Phys. Rev. Lett., 64 [22] 2727-30 (1990).

${ }^{26}$ B. J. Buchalter and R. M. Bradley, "Orientational Order in Amorphous Packings of Ellipsoids," Europhys. Lett., 26 [3] 159-64 (1994).

${ }^{27}$ D. Coelho, J. F. Thovert, and P. M. Adler, "Geometrical and Transport Properties of Random Packings of Spheres and Aspherical Particles," Phys. Rev. E, 55 [2] 1959-78 (1997).

${ }^{28}$ F. M. Borodich, L. M. Keer, and C. S. Korach, "Analytical Study of Fundamental Nanoindentation Test Relations for Indenters of Non-Ideal Shapes," Nanotechnology, 14 [7] 803-8 (2003).

${ }^{29}$ F. P. Ganneau, G. Constantinides, and F.-J. Ulm, "Dual-Indentation Technique for the Assessment of Strength Properties of Cohesive-Frictional Materials," Int. J. Solids Struct., 43 [6] 1727-45 (2006).

${ }^{30} \mathrm{C}$. Hellmich and F.-J. Ulm, "Are Mineralized Tissues Open Crystal Foams Reinforced by Crosslinked Collagen?-Some Energy Arguments," J. Biomech., 35 [9] 1199-212 (2002).

${ }^{31}$ C. Hellmich and F.-J. Ulm, "Micromechanical Model for Ultrastructural Stiffness of Mineralized Tissues," J. Eng. Mech.-ASCE, 128 [8] 898-908 (2002).

${ }^{32}$ A. J. Allen, J. J. Thomas, and H. M. Jennings, "Composition and Density of Nanoscale Calcium-Silicate-Hydrate in Cement," Nat. Mater., 6, 311-6 (2007).

${ }^{33}$ R. J. M. Pellenq and H. Van Damme, "Why Does Concrete Set?: The Nature of Cohesion Forces in Hardened Cement-Based Materials," MRS Bull., 29 [5] 319-23.

${ }^{34}$ J. J. Chen, J. J. Thomas, H. F. W. Taylor, and H. M. Jennings, "Solubility and Structure of Calcium Silicate Hydrate," Cement Concrete Res., 34 [9] 1499-51 (2004)

${ }^{35}$ K. Velez, S. Maximilien, D. Damidot, G. Fantozzi, and F. Sorrentino, "Determination by Nanoindentation of Elastic Modulus and Hardness of Pure Constituents of Portland Cement Clinker," Cement Concrete Res., 31 [4] 555-61 (2001).

${ }^{36}$ H. M. Jaeger and S. R. Nagel, "Physics of Granular State," Science, 255 [5051] 1523-31 (1992)

${ }^{37}$ N. J. A. Sloane, “Kepler's Conjecture Confirmed," Nature, 395, 435-6 (1998).

${ }^{38}$ A. Donev, I. Cisse, D. Sachs, E. A. Variano, F. H. Stillinger, R. Connely, S. Torquato, and P. M. Chaikin, "Improving the Density of Jammed Disordered Packings Using Ellipsoids," Science, 303, 990-3 (2004).

${ }^{39}$ S. Diamond, "Mercury Porosimetry-An Inappropriate Method for the Measurement of Pore Size Distributions in Cement-Based Materials," Cement Concrete Res., 30 [10] 1517-25 (2000).

${ }^{40}$ K. S. Prostak and S. Lees, "Visualization of Crystal-Matrix Structure. In situ Demineralization of Mineralized Turkey leg Tendon and Bone," Calcif. Tissue Int., 59 [6] 474-9 (1996).

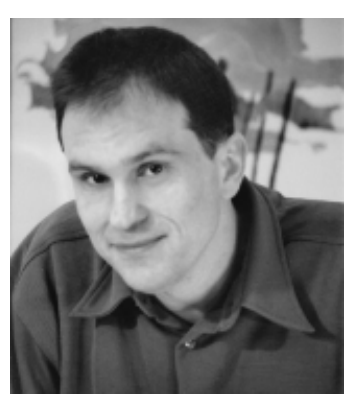

Franz-Josef Ulm is a Professor of Civil \& Environmental Engineering at the Massachusetts Institute of Technology. He received his engineering degree from the Technical University Munich, Germany, in 1990, his Ph.D. from the Ecole nationale des ponts et chaussées, France, in 1994; and his Habilitation Degree from the Ecole Normale Supérieure de Cachan, France in 1998. Before joining MIT, he was research engineer at the Laboratoire Central des Ponts et Chaussées of the French Department of Transportation and Public Works. He joined MIT in January 1999, where he is responsible for mechanics of materials, with a current focus on experimental and theoretical nanomechanics of natural composites (concrete, soil, rock, bone), microporomechanics and biochemomechanics. He has published over 80 scientific papers in peer-reviewed mechanics, materials science, and biomechanics
${ }^{41}$ J. D. Currey, "The Relationship Between the Stiffness and the Mineral Content of Bone," J. Biomech., 2, 477-80 (1969).

${ }^{42}$ H. J. Gao, B. H. Ji, I. L. Jager, I. Arzt, and P. Fratzl, "Materials Become Insensitive to Flaws at Nanoscale: Lessons from Nature," Proc. Natl. Acad. Sci. USAm, 100 [10] 5597-600 (2003).

${ }^{43}$ M. A. Rubin, L. Jasiuk, J. Taylor, J. Rubin, T. Ganey, and R. P. Apkarian, "TEM Analysis of the Nanostructure of Normal and Osteoporotic Human Trabecular Bone," Bone, 33 [3] 270-82 (2003).

${ }^{44}$ V. B. Rosen, L. W. Hobbs, and M. Spector, "The Ultrastructure of Anorganic Bovine Bone and Selected Synthetic Hyroxyapatites Used as Bone Graft Substitute Materials," Biomaterials, 23 [3] 921-8 (2002).

${ }^{45}$ J. Catanese, J. D. B. Featherstone, and T. M. Keaveny, "Characterization of the Mechanical and Ultrastructural Properties of Heat-Treated Cortical Bone for Use as a Bone Substitute," J. Biomed. Mater. Res., 45 [4] 327-36 (1999).

${ }^{46}$ K. S. Tai, H. J. Qi, and C. Ortiz, "Effect of Mineral Content on the Nanoindentation Properties and Nanoscale Deformation Mechanisms of Bovine Tibial Cortical Bone,” J. Mater. Sci.-Mater. Med., 16 [10] 947-59 (2005).

${ }^{47}$ S. Lees and F. R. Rollins, "Anisotropy in Hard Dental Tissues," J. Biomech., 5, 557-66 (1972)

${ }^{48}$ S. Lees, D. Hanson, and E. A. Page, "Some Acoustical Properties of the Otic Bones of a Fin Whale," J. Acoust. Soc. Am., 99 [4] 2421-7 (1995).

${ }^{49}$ R. S. Gilmore and J. L. Katz, "Elastic Properties of Apatites," J. Mater. Sci., 17 [4] 1131-4 (1982)

${ }^{50} \mathrm{C}$. Hellmich, J. F. Barthelemy, and L. Dormieux, "Mineral-Collagen Interactions in Elasticity of Bone Ultrastructure-A Continuum Micromechanics Approach," Eur. J. Mech. A-Solids, 23 [5] 783-810 (2004).

${ }^{51}$ F.-J. Ulm, A. Delafargue, and G. Constantinides, "Experimental Microporomechanics"; pp. 207-88 in Applied Micromechanics of Porous Materials. CISM Lecture Notes No. 580, Edited by L. Dormieux, and F. -J. Ulm. Springer Wien, New York, 2005.

${ }^{52}$ E. A. Kaarsberg, "Introductory Studies of Natural and Artificial Argillaceous Aggregates by Sound-Propagation and X-Ray Diffraction Methods," J. Geol., 67, 447-72 (1959)

${ }^{53}$ B. Hornby, L. Schwartz, and J. Hudson, "Anisotropic Effective Medium Modeling of the Elastic Properties of Shales," Geophysics, 59 [10] 1570-83 (1994).

${ }^{54}$ C. M. Sayers, "The Elastic Anisotropy of Shales," J. Geophys. Res., 99 [B1] 767-74 (1994).

${ }^{55}$ M. S. Bobji and S. K. Biswas, "Deconvolution of Hardness from Data Obtained from Nanoindentation of Rough Surfaces," J. Mater. Res., 14 [6] 2259-68 (1998).

${ }^{56}$ E. Donnelly, S. P. Baker, A. L. Boskey, and M. C. H. van der Meulen, "Effects of Surface Roughness and Maximum Load on the Mechanical Properties of Cancellous Bone Measured by Nanoindentation," J. Biomed. Mater. Res. A, 77 [2] 426-35 (2006).

${ }^{57}$ J. A. Ortega, F.-J. Ulm, and Y. Abousleiman, "The Effect of the Nanogranular Nature of Shale on their Poroelastic Properties," Acta Geotechn., 2007, in press.

${ }^{58}$ A. V. Hershey, "The Elasticity of an Isotropic Aggregate of Anisotropic Cubic Crystals," ASME J. Appl. Mech., 21, 236-40 (1954).

${ }^{59}$ E. Kröner, "Berechnung der elastischen Konstanten des Vielkristalls aus den Konstanten des Einkristalls," Z. Phys., 151, 504-18 (1958).

${ }^{60}$ R. Hill, "A Self-Consistent Mechanics of Composite Materials," J. Mech. Phys. Solids, 13, 213-22 (1965).

${ }^{61}$ B. Budiansky, "On the Elastic Moduli of Some Heterogeneous Materials," J. Mech. Phys. Solids, 13, 223-7 (1965).

${ }^{62}$ E. Kröner, "Self-Consistent Scheme and Graded Disorder in Polycrystal Elasticity," J. Phys. F: Met. Phys., 8, 2261-7 (1978).

${ }^{63}$ N. Laws, "A Note of Penny-Shaped Cracks in Transversely Isotropic Materials," Mech. Mat., 4, 209-12 (1985).

journals, and four books; most recently "Microporomechanics" (co-authored with L. Dormieux and D. Kondo) published by John Wiley \& Sons in 2006.

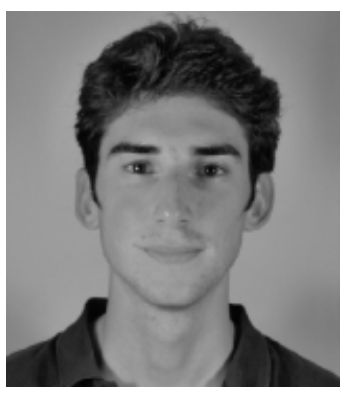

Matthieu Vandamme is a Ph.D. candidate in the Department of Civil and Environmental Engineering at the Massachusetts Institute of Technology. He graduated from Ecole Polytechnique, France, in 2002; and received his M.S. in Civil Engineering from Ecole nationale des ponts et chaussées, France, in 2003, where he worked on the modeling of piezoelectric composite materials. He is Civil Servant of the French Ministry of Ecology, Sustainable Building and Development. His Ph.D. research focuses on the nanoindentation characterization of microstructure and viscous properties of cementitious materials. 


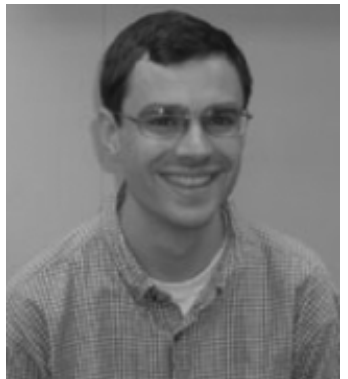

Christopher P. Bobko is a Ph.D. candidate in the Department of Civil and Environmental Engineering at the Massachusetts Institute of Technology. He received his B.S. degree in Civil and Environmental Engineering from Princeton University in 2003, and his S.M. degree in Civil and Environmental Engineering from MIT. His Ph.D. research focuses on the development of nanoindentation techniques for the assessment of properties of nanogranular materials in general, and shale materials in particular.

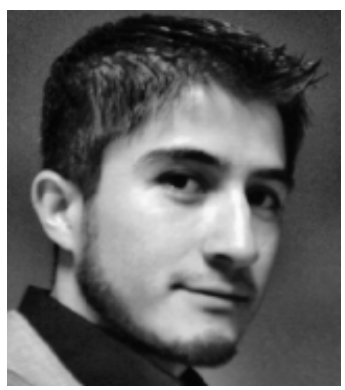

J. Alberto Ortega is a Ph.D. candidate in the Department of Civil and Environmental Engineering at the Massachusetts Institute of Technology. He received his B.S. degree in Civil Engineering from Texas A\&M University and his S.M degree in Civil and Environmental Engineering from MIT. His Ph.D. research focuses on the nanomechanics of shale, the multi-scale modeling of heterogeneous materials, the characterization of seismic anisotropy in sedimentary rocks, and the micromechanics of porous media.

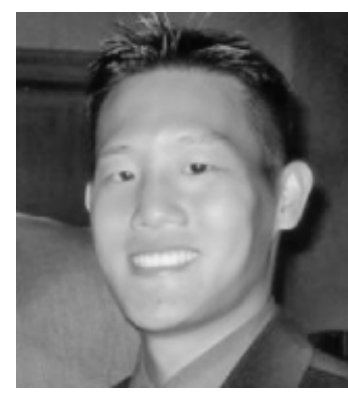

Kuangshin Tai received his Ph.D. from the Department of Materials Science and Engineering at the Massachusetts Institute of Technology, and received his B.S. degree in Bioengineering with a concentration in Mechanics and Materials from the University of California at Berkeley. His Ph.D. research focused on the ultrastructure and underlying nanomechanics in bone as related to nanoscale heterogeneity, energy dissipation, mechanisms of deformation, and cohesivefrictional behavior. He was chosen as a Graduate Student Award Medal Winner for the Materials Research Society and was also a Whitaker Fellowship Recipient for Biomedical Engineering Research. Dr. Tai currently works as a Technology Specialist for Wolf, Greenfield, and Sacks, an Intellectual Property Law Firm in Boston, MA

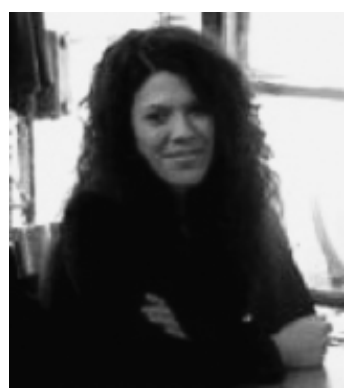

Professor Christine Ortiz obtained her B.S. from Rensselaer Polytechnic Institute in Troy, NY and her M.S. and Ph.D. from Cornell University in Ithaca, NY, all in the field of materials science and engineering. After graduation, Professor Ortiz was granted a NSFNATO post-doctoral fellowship and moved to the Department of Polymer Chemistry, University of Groningen, in the Netherlands. Professor Ortiz joined the faculty in the Department of Materials Science and Engineering at MIT as an Assistant Professor in 1999 and was tenured in 2006. Her research group focuses on the ultrastructure and nanomechanics of structural biological materials such as cartilage, bone, seashells, and armored fish. In 2002, Dr. Ortiz was awarded a National Science Foundation Presidential Early Career Award for Scientists and Engineers (NSF-PECASE) which was presented to her by President George W. Bush at the White House in Washington DC. In 2007, Professor Ortiz was nominated and selected to participate in the 2008-2009 Defense Science Study Group. Professor Ortiz has a strong commitment to teaching, mentoring, and increasing diversity at all educational levels. 